QUARTERLY OF APPLIED MATHEMATICS

VOLUME LXX, NUMBER 3

SEPTEMBER 2012, PAGES 437-467

S 0033-569X(2012)01325-0

Article electronically published on May 25, 2012

\title{
THE ASYMPTOTICS OF HEAVILY BURDENED VISCOELASTIC RODS
}

\author{
BY
}

STUART S. ANTMAN (Department of Mathematics, Institute for Physical Science and Technology, and Institute for Systems Research, University of Maryland, College Park, Maryland 20742)

AND

SÜLEYMAN ULUSOY (Faculty of Education, Zirve University, Kızlhisar Campus, 27260

Gaziantep, Turkey)

This paper is dedicated to Costas Dafermos on the occasion of his 70th birthday.

\begin{abstract}
This paper treats the spatial motion of a deformable nonlinearly viscoelastic rod carrying a heavy rigid body. The ratio of the inertia of the rod to that of the attached rigid body is characterized by a small parameter $\varepsilon$. The boundary conditions on the rod where it is attached to the rigid body are the ordinary differential equations of motion for the rigid body subject to the contact loads exerted on the rigid body by the rod. The entire system is thus governed by a quasilinear parabolic-hyperbolic system of partial differential equations coupled to the ordinary differential equations for the rigid body, with $\varepsilon$ appearing in the coefficients of the acceleration terms of the rod. This paper gives a rigorous asymptotic expansion of the solutions of initial-boundary-value problems for this system, consisting of a regular expansion and an initial-layer expansion. The leading term of the regular expansion satisfies the reduced problem, obtained by setting $\varepsilon=0$ in the governing equations. The reduced problem is governed by a curious set of quasilinear functional-differential equations, the solutions of which exhibit a rich and interesting behavior. (In the absence of dissipation, which is needed for the justification of the asymptotic expansion, the leading term of the regular expansion satisfies a steady-state problem parametrized by time, which enters through the boundary conditions.) The remaining terms of the regular expansion satisfy linear problems. The leading term of the initial-layer expansion satisfies a quasilinear parabolic system, and the remaining terms satisfy linear parabolic systems. Thus the asymptotic expansion leads to greatly simplified equations. The regular and initial-layer corrections to the
\end{abstract}

Received January 2, 2012.

2010 Mathematics Subject Classification. Primary 35B25, 35C20, 35G31, 35K35, 35K70, 74C20, 74D10,74K10.

E-mail address: ssa@math.umd.edu

E-mail address: suleyman.ulusoy@zirve.edu.tr 
solution of the reduced problem show that it exhibits the main features of the solution to the whole system.

The justification of the asymptotic expansion consists in estimating the error. For this purpose, a Faedo-Galerkin method is used to obtain sharp estimates for the exponential decay in time of the terms of the initial-layer expansion (satisfying parabolic systems). (This method is far more efficient than the repeated use of the Maximum Principle à la S. N. Bernstein (see Wiegner, Math. Z. 188 (1984) 3-22) for treating the analogous scalar problem by Yip et al., J. Math. Pures Appl. 81 (2002) 283-309. The Maximum Principle is not applicable to our parabolic systems. Even for such scalar problems, the Faedo-Galerkin method as used here is far simpler and more efficient.) The main focus of this paper is on the derivation of these estimates.

A significant part of the analysis is devoted to handling technical difficulties due to the peculiarities of the geometrically exact equations governing the spatial motion of viscoelastic rods with a general class of nonlinear constitutive equations of strain-rate type invariant under rigid motions.

1. Introduction. The simplest problem for the mechanics of deformable bodies is to determine the motion of a mass point on a spring, in which the spring, regarded as massless, is merely a device to communicate a force to the mass point depending solely on the position and velocity of the mass point, so that its motion is governed by a scalar second-order ordinary differential equation. If the spring is reckoned to have small mass, however, and is accordingly governed by a partial differential equation accounting for its material response, then a rigorous asymptotic expansion of the solutions in terms of a small density parameter $\varepsilon$ for the spring is possible when there is a sufficiently strong internal dissipation 21. This analysis gives a precise status to the reduced problem, obtained by setting $\varepsilon=0$. This reduced problem is not governed by an ordinary differential equation [1, 5]; its equations exhibit memory effects. The same remarks apply to other motions of a rigid body with one degree of freedom, such as those produced by the shearing or twisting of a light deformable body to which it is attached [20]. (In a series of papers beginning with [7, Beatty treated such simple motions, governed by scalar second-order ordinary differential equations, of inertialess deformable solids carrying rigid bodies, with the effects of the deformable body on the rigid body inherited from constitutive equations for 3-dimensional bodies.) The limited role of ordinary differential equations as attractors for such problems with the deformable solid having small inertia was treated in [18, 19, 20]. (In 1911, Timoshenko [22, Sec. 4.9] produced a recipe of limited generality to account for the mass of a spring by a scalar second-order ordinary differential equation.)

If, however, the motion of the deformable body requires more than a single scalarvalued function to describe it, as for the problem of a bob on a deformable pendulum shaft, then the behavior of solutions of the governing equations is far more complicated than that for longitudinal motion of a mass point on a spring. This complexity is exposed in the treatment [3] of the reduced problem for the spatial motion of a rigid body on a light viscoelastic rod, which shows that solutions typically cannot be governed by ordinary differential equations. The text 22 and many others, in stating that motions 
like those of a bob on a deformable pendulum shaft, an arrow in a bow, and a mass point on an extensible string can be described by scalar second-order ordinary differential equations, tacitly assume that the motion of the rigid body is severely constrained and the motion of the deformable body possesses a uniformity for which there is no physical warrant.

Here we extend the treatment 3 ] of the reduced problem for the spatial motion of a rigid body on a light viscoelastic rod to the full asymptotics, which gives a precise position to the reduced problem with its curious behavior. The justification of the asymptotics consists in estimating the error, which relies on sharp estimates for the exponential decay in time of the terms of the initial-layer expansion. Because we have a vectorial system, the derivation of these estimates as in 21] by the repeated use of the Maximum Principle à la S. N. Bernstein [17] is not available. Instead, we use a far simpler and more efficient Faedo-Galerkin method. We focus much of our attention on constructing these estimates in Section 8 Our main source of difficulty is the mathematical structure of the geometrically exact equations of motion, for which the constitutive equations must be invariant under rigid motions. (The paper [1] has some of the flavor of the problem we treat, although its aim and methods are completely different. The role of the small parameter in [10] is the opposite of that here.)

Notation. We employ Gibbs notation for vectors and tensors: Vectors, which are elements of Euclidean 3 -space $\mathbb{E}^{3}$, and vector-valued functions are denoted by lower-case, italic, bold-face symbols. The dot product and cross product of (vectors) $\boldsymbol{u}$ and $\boldsymbol{v}$ are denoted $\boldsymbol{u} \cdot \boldsymbol{v}$ and $\boldsymbol{u} \times \boldsymbol{v}$. In our work, a tensor is just a linear transformation from $\mathbb{E}^{3}$ to itself. The value of tensor $\boldsymbol{A}$ at vector $\boldsymbol{v}$ is denoted $\boldsymbol{A} \cdot \boldsymbol{v}$ (in place of the more usual $\boldsymbol{A} \boldsymbol{v}$ ). The product of tensors $\boldsymbol{A}$ and $\boldsymbol{B}$ is denoted $\boldsymbol{A} \cdot \boldsymbol{B}$ (in place of the more usual $\boldsymbol{A} \boldsymbol{B}$ ). The transpose of $\boldsymbol{A}$ is denoted $\boldsymbol{A}^{\top}$. We write $\boldsymbol{v} \cdot \boldsymbol{A}=\boldsymbol{A}^{\top} \cdot \boldsymbol{v}$. The dyadic product of vectors $\boldsymbol{a}$ and $\boldsymbol{b}$ is the tensor denoted $\boldsymbol{a} \boldsymbol{b}$ (in place of the more usual $\boldsymbol{a} \otimes \boldsymbol{b}$ ), which is defined by $(\boldsymbol{a b}) \cdot \boldsymbol{v}=(\boldsymbol{b} \cdot \boldsymbol{v}) \boldsymbol{a}$ for all $\boldsymbol{v}$. (A dyadic product is a tensor of rank 1.) The transpose of $\boldsymbol{a} \boldsymbol{b}$ is $(\boldsymbol{a b})^{\top}=\boldsymbol{b} \boldsymbol{a}$. Since a suitable set of nine dyadic products forms a basis for the space of tensors, we can define all the properties of tensors by defining them merely for dyadic products. We accordingly define $\boldsymbol{u} \times \boldsymbol{A}$ by $\boldsymbol{u} \times(\boldsymbol{a} \boldsymbol{b}):=(\boldsymbol{u} \times \boldsymbol{a}) \boldsymbol{b}$.

Triples of real numbers, such as $\mathbf{u} \equiv\left(u_{1}, u_{2}, u_{3}\right)$, which are elements of $\mathbb{R}^{3}$, are denoted by lower-case, sans-serif, bold-face letters. $\mathbb{R}^{3}$ is endowed with the usual dot and cross products defined componentwise. The difference between it and $\mathbb{E}^{3}$ is that elements of $\mathbb{R}^{3}$ need have no natural geometrical or physical significance.

The (Gâteaux) differential of $\boldsymbol{u} \mapsto \boldsymbol{f}(\boldsymbol{u})$ at $\boldsymbol{v}$ in the direction $\boldsymbol{h}$ is $\left.\frac{d}{d \xi} \boldsymbol{f}(\boldsymbol{v}+\xi \boldsymbol{h})\right|_{\xi=0}$. When it is linear in $\boldsymbol{h}$, we denote this differential by $\frac{\partial \boldsymbol{f}}{\partial \boldsymbol{u}}(\boldsymbol{v}) \cdot \boldsymbol{h}$ or $\boldsymbol{f}_{\boldsymbol{u}}(\boldsymbol{v}) \cdot \boldsymbol{h}$. We denote the derivative of a function $\boldsymbol{f}$ with respect to a scalar argument $\tau$ by $\boldsymbol{f}_{\tau}$ or $\partial_{\tau} \boldsymbol{f}$. We often denote a function like $\boldsymbol{u} \mapsto \boldsymbol{f}(\boldsymbol{u}, \boldsymbol{v})$ by $\boldsymbol{f}(\cdot, \boldsymbol{v})$.

2. Formulation of the governing equations. We give a précis of the governing equations in a form somewhat different from that derived in detail in [3], closely following several paragraphs (cf. [2, Chap. 8]). In this section, we use the summation convention that twice-repeated lower-case italic indices other than $s$ and $t$ are summed from 1 to 3 . 
Kinematics. The motion of a rod that can suffer flexure, extension, torsion, and shear is defined by three vector-valued functions

$$
[0,1] \times[0, \infty) \ni(s, t) \mapsto \boldsymbol{r}(s, t), \boldsymbol{d}_{1}(s, t), \boldsymbol{d}_{2}(s, t) \in \mathbb{E}^{3}
$$

with $\boldsymbol{d}_{1}(s, t)$ and $\boldsymbol{d}_{2}(s, t)$ orthonormal. The curve $\boldsymbol{r}(\cdot, t)$ may be interpreted as the configuration at time $t$ of any material curve connecting the "ends" of a slender 3 -dimensional body, e.g., a curve of centroids of the cross sections. $\boldsymbol{d}_{1}(s, t)$ and $\boldsymbol{d}_{2}(s, t)$ may be interpreted as characterizing the configuration of the material section (at) $s$ at time $t$. We assume that $s$ is the arc-length parameter of the reference configuration of $\boldsymbol{r}$ scaled so that $0 \leq s \leq 1$. We set

$$
\boldsymbol{d}_{3} \equiv \boldsymbol{d}_{1} \times \boldsymbol{d}_{2}
$$

so that for each $(s, t),\left\{\boldsymbol{d}_{k}(s, t)\right\}$ is a right-handed orthonormal basis for $\mathbb{E}^{3}$. This orthonormality implies that there are vector-valued functions $\boldsymbol{u}$ and $\boldsymbol{w}$ such that

$$
\partial_{s} \boldsymbol{d}_{k}=\boldsymbol{u} \times \boldsymbol{d}_{k}, \quad \partial_{t} \boldsymbol{d}_{k}=\boldsymbol{w} \times \boldsymbol{d}_{k} .
$$

Let $\left\{\mathbf{e}_{1}, \mathbf{e}_{2}, \mathbf{e}_{3}\right\}$ be the standard basis for $\mathbb{R}^{3}$. Then the proper-orthogonal linear transformation taking the orthonormal basis $\left\{\mathbf{e}_{k}\right\}$ to the right-handed orthonormal basis $\left\{\boldsymbol{d}_{k}(s, t)\right\}$ is

$$
\boldsymbol{D}(s, t):=\boldsymbol{d}_{k}(s, t) \mathbf{e}_{k} \quad\left(\Longleftrightarrow \quad \boldsymbol{d}_{k}=\boldsymbol{D} \cdot \mathbf{e}_{k}, \quad \boldsymbol{D}^{-1}=\mathbf{e}_{k} \boldsymbol{d}_{k}=: \mathbf{D}^{\top}\right) .
$$

Thus

$$
\boldsymbol{D}_{s}=\boldsymbol{u} \times \boldsymbol{D}, \quad \boldsymbol{D}_{t}=\boldsymbol{w} \times \boldsymbol{D} .
$$

For our asymptotic analysis, we use the rotation tensor $\boldsymbol{D}$ in preference to the basis $\left\{\boldsymbol{d}_{k}\right\}$ because $\boldsymbol{D}$ lacks subscripts that would further clutter up those unavoidable in the asymptotic analysis.

We decompose relevant vector-valued functions with respect to the natural basis $\left\{\boldsymbol{d}_{k}\right\}$ :

$$
\boldsymbol{r}_{s}=: \boldsymbol{v}=v_{k} \boldsymbol{d}_{k}, \quad \boldsymbol{u}=u_{k} \boldsymbol{d}_{k}, \quad \boldsymbol{w}=w_{k} \boldsymbol{d}_{k} .
$$

For any vector $\boldsymbol{z}=z_{k} \boldsymbol{d}_{k}$ we define the triples $\left(z_{1}, z_{2}, z_{3}\right)$ of its components with respect to the basis $\left\{\boldsymbol{d}_{k}\right\}$ by

$$
\mathbf{z} \equiv\left(z_{1}, z_{2}, z_{3}\right) \equiv z_{k} \mathbf{e}_{k} \equiv \mathbf{D}^{\top} \cdot \boldsymbol{z}=: \boldsymbol{z} \cdot \boldsymbol{D}
$$

The triples

$$
\mathbf{v} \equiv\left(v_{1}, v_{2}, v_{3}\right) \equiv \mathbf{D}^{\top} \cdot \boldsymbol{v}, \quad \mathbf{u} \equiv\left(u_{1}, u_{2}, u_{3}\right) \equiv \mathbf{D}^{\top} \cdot \boldsymbol{u}
$$

are the strain variables for the motion (2.1). They satisfy

$$
\boldsymbol{v}=\boldsymbol{D} \cdot \mathbf{v}, \quad \boldsymbol{u}=\boldsymbol{D} \cdot \mathbf{u}, \quad \boldsymbol{v}_{t}=\boldsymbol{D} \cdot \mathbf{v}_{t}+(\boldsymbol{w} \times \boldsymbol{D}) \cdot \mathbf{v}, \quad \boldsymbol{u}_{t}=\boldsymbol{D} \cdot \mathbf{u}_{t}+(\boldsymbol{w} \times \boldsymbol{D}) \cdot \mathbf{u} .
$$

Note, however, that

$$
\boldsymbol{w}_{t}=\boldsymbol{D} \cdot \mathbf{w}_{t}
$$

$\boldsymbol{D}$ satisfies the identities

$$
(\boldsymbol{D} \cdot \mathbf{a}) \cdot(\boldsymbol{D} \cdot \mathbf{b})=\mathbf{a} \cdot \mathbf{b}, \quad(\boldsymbol{D} \cdot \mathbf{a}) \times(\boldsymbol{D} \cdot \mathbf{b})=\boldsymbol{D} \cdot(\mathbf{a} \times \mathbf{b}) .
$$

The equality of mixed partial derivatives of the $\boldsymbol{d}_{k}$ implies the compatibility condition

$$
\boldsymbol{w}_{s}=\boldsymbol{u}_{t}+\boldsymbol{u} \times \boldsymbol{w}=\left(\partial_{t} u_{k}\right) \boldsymbol{d}_{k}=\boldsymbol{D} \cdot \mathbf{u}_{t} .
$$


For fixed $t$ the functions $\mathbf{u}(\cdot, t)$ and $\mathbf{v}(\cdot, t)$ determine $\boldsymbol{r}(\cdot, t), \boldsymbol{d}_{1}(\cdot, t), \boldsymbol{d}_{2}(\cdot, t)$ (the configuration at time $t$ ) to within a rigid motion. The strains $u_{1}$ and $u_{2}$ measure flexure, $u_{3}$ measures torsion, $v_{1}$ and $v_{2}$ measure shear, and $v_{3}$ measures dilatation.

A rod-theoretic analog of the 3-dimensional requirement that the Jacobian of the deformation be positive (so that orientation is preserved) is that there be a function $\left(u_{1}, u_{2}, s\right) \mapsto V\left(u_{1}, u_{2}, s\right)$ for which $V(0,0, s)=0, V\left(u_{1}, u_{2}, s\right)>0$ for $u_{\alpha} u_{\alpha}>0$, and $V(\cdot, \cdot, s)$ is convex and homogeneous of degree 1 such that

$$
v_{3}>V\left(u_{1}, u_{2}, s\right) \text {. }
$$

By imposing appropriate physically natural (but not so obvious) constitutive restrictions, we can ensure that no solution of the initial-boundary-value problems we consider can violate this condition for any finite time [4]. Since the asymptotic representation of the solution that we obtain is only valid for any preassigned finite time, we do not bother to impose such restrictions. Likewise we restrict our attention to motions that have no self-contact or that terminate before such self-contact occurs.

Let $\left\{\boldsymbol{i}_{1}, \boldsymbol{i}_{2}, \boldsymbol{i}_{3}\right\}$ be a fixed right-handed orthonormal basis for $\mathbb{E}^{3}$. To be specific, we assume that the end $s=0$ of the rod is welded to the $\left\{\boldsymbol{i}_{1}, \boldsymbol{i}_{2}\right\}$-plane at the origin, so that

$$
\boldsymbol{r}(0, t)=\boldsymbol{o}, \quad \boldsymbol{d}_{k}(0, t)=\boldsymbol{i}_{k} \quad \Longleftrightarrow \boldsymbol{D}(0, t)=\boldsymbol{i}_{k} \mathbf{e}_{k} .
$$

(We are effectively identifying the basis $\left\{\mathbf{e}_{k}\right\}$ with the basis $\left\{\boldsymbol{i}_{k}\right\}$.) We assume that the rigid body is rigidly attached to the other end $s=1$ of the rod. Its mass center is at $\boldsymbol{r}(1, t)+\boldsymbol{c}(t)$, its mass is $\mu$, and its moment of inertia tensor about the mass center in the reference configuration is the given constant $\mathbf{J}:=J_{k l} \mathbf{e}_{k} \mathbf{e}_{l}$. Its moment of inertia tensor about the mass center in the configuration at time $t$ is thus

$$
\boldsymbol{J}(t):=J_{k l} \boldsymbol{d}_{k}(1, t) \boldsymbol{d}_{l}(1, t)=J_{k l} \boldsymbol{D}(1, t) \cdot \mathbf{e}_{k} \boldsymbol{D}(1, t) \cdot \mathbf{e}_{l}=\boldsymbol{D}(1, t) \cdot \mathbf{J} \cdot \mathbf{D}(1, t)^{\top} .
$$

Since the rigid body is rigidly attached to the rod,

$$
\boldsymbol{c}(t)=c_{k} \boldsymbol{d}_{k}(1, t)=\boldsymbol{D}(1, t) \cdot \mathbf{c}
$$

where the $\mathbf{c}=\left(c_{1}, c_{2}, c_{3}\right)$ is a given constant in $\mathbb{R}^{3}$. The angular momentum of the rigid body about its mass center is

$$
\boldsymbol{J}(t) \cdot \boldsymbol{w}(1, t) \equiv \boldsymbol{D}(1, t) \cdot \mathbf{J} \cdot \mathbf{D}(1, t)^{\top} \cdot \boldsymbol{D}(1, t) \cdot \mathbf{w}(1, t) \equiv \boldsymbol{D}(1, t) \cdot \mathbf{J} \cdot \mathbf{w}(1, t) .
$$

Thus both $\boldsymbol{c}$ and the orientation of the rigid body are determined by the $\boldsymbol{D}(1, t)$.

The linear momentum and angular momentum of the rod per unit reference length at $(s, t)$ are taken to have the scaled forms

$$
\begin{gathered}
\varepsilon(\rho A)(s) \boldsymbol{r}_{t}(s, t), \\
\chi(\varepsilon)(\rho \boldsymbol{I})(s, t) \cdot \boldsymbol{w}(s, t) \equiv \chi(\varepsilon)\left(\rho I_{p q}\right)(s) w_{q}(s, t) \boldsymbol{d}_{p}(s, t) \equiv \chi(\varepsilon) \boldsymbol{D}(s, t) \cdot(\rho \mathbf{I})(s) \cdot \mathbf{w}(s, t)
\end{gathered}
$$

where $\varepsilon$ is a small nonnegative parameter, $\chi$ is a small prescribed function with $\chi(\varepsilon)>0$ for $\varepsilon>0$, and with $\chi(0)=0, \rho A$ is a prescribed positive-valued function and the $\left(\rho I_{\gamma \delta}\right)(s)$ are prescribed components of a positive-definite symmetric $3 \times 3$ matrix $(\rho \mathbf{I})(s) \equiv$ 
$\left(\rho I_{p q}\right)(s) \mathbf{e}_{p} \mathbf{e}_{q}$, with $\rho I_{\gamma 3}=\rho I_{3 \gamma}=0, \rho I_{33}=\rho I_{\gamma \gamma}$, and $\rho \boldsymbol{I} \equiv \rho I_{p q} \boldsymbol{d}_{p} \boldsymbol{d}_{q} \equiv \boldsymbol{D} \cdot \rho \mathbf{I} \cdot \mathbf{D}^{\top}$. We assume that there are positive constants $c$ and $C$ such that

$$
c \leq \rho A \leq C, \quad c|\mathbf{a}|^{2} \leq \mathbf{a} \cdot \mathbf{l} \cdot \mathbf{a} \leq C|\mathbf{a}|^{2} \quad \forall \mathbf{a} .
$$

(If $\rho \mathbf{I}=\mathbf{O}$, then we would have to require that $\rho \mathbf{J}=\mathbf{O}$. In this case, the problem would become that for a string, which would be easier to analyze except when parts of it are under compression.)

The parameter $\varepsilon$ is interpreted as the ratio of the actual mass of the rod (in the unscaled formulation) to that of the rigid body at its end, and $\chi(\varepsilon)$ may be interpreted as a ratio of a norm of the moment of inertia matrix for the rod about its mass center in the reference configuration to a norm of the moment of inertia tensor of the rigid body about its mass center. More generally, we could replace $\varepsilon(\rho A)(s)$ with $(\rho A)(s, \varepsilon)$ and replace $\chi(\varepsilon)(\rho \mathbf{I})(s)$ with $(\rho \mathbf{I})(s, \varepsilon)$ to account for a strongly varied mass and inertia distribution along the rod. To be specific and to minimize unilluminating complications, however, we limit our attention to (2.18) with

$$
\chi(\varepsilon)=\varepsilon .
$$

(Our methods would immediately handle the case that $\chi(\varepsilon)$ has a positive-valued finite Taylor expansion in $\varepsilon$ beginning with $\varepsilon$, and variants of our methods would handle other possibilities.)

Equations of motion. In the configuration at time $t$, the resultant internal contact force and internal contact couple exerted by the material of $[0, s)$ on the material of $[s, 1]$ (for $0<s \leq 1$ ) are respectively denoted $-\boldsymbol{n}(s, t)$ and $-\boldsymbol{m}(s, t)$. For simplicity of exposition, we assume that there is neither body force nor body couple.

The equations of motion for the rod and the rigid attachment are

$$
\begin{gathered}
\boldsymbol{n}_{s}=\varepsilon(\rho A)(s) \boldsymbol{r}_{t t} \\
\boldsymbol{m}_{s}+\boldsymbol{r}_{s} \times \boldsymbol{n}=\varepsilon \partial_{t}[(\rho \boldsymbol{I})(s, t) \cdot \boldsymbol{w}(s, t)] \equiv \varepsilon \partial_{t}[\boldsymbol{D}(s, t) \cdot(\rho \mathbf{I})(s) \cdot \mathbf{w}(s, t)], \\
-\boldsymbol{n}(1, t)=\mu\left[\boldsymbol{r}_{t t}(1, t)+\boldsymbol{c}_{t t}(t)\right] \\
-\boldsymbol{m}(1, t)+\boldsymbol{c}(t) \times \boldsymbol{n}(1, t)=[\boldsymbol{J}(t) \cdot \boldsymbol{w}(1, t)]_{t} \equiv[\boldsymbol{D}(1, t) \cdot \mathbf{J} \cdot \mathbf{w}(1, t)]_{t} .
\end{gathered}
$$

Note that

$$
\begin{aligned}
\boldsymbol{c}_{t}(t) & =\boldsymbol{w}(1, t) \times \boldsymbol{c}(t) \equiv[\boldsymbol{w}(1, t) \times \boldsymbol{D}(1, t)] \cdot \mathbf{c}, \\
\boldsymbol{w}_{t}(1, t) & =\partial_{t} w_{k}(1, t) \boldsymbol{d}_{k}(1, t) \equiv \boldsymbol{D}(1, t) \cdot \mathbf{w}_{t}(1, t), \\
\boldsymbol{c}_{t t}(t) & =\boldsymbol{w}_{t}(1, t) \times \boldsymbol{c}(t)+\boldsymbol{w}(1, t) \times[\boldsymbol{w}(1, t) \times \boldsymbol{c}(t)] \\
& \equiv\left\{\boldsymbol{w}_{t}(1, t) \times \boldsymbol{D}(1, t)+\boldsymbol{w}(1, t) \times[\boldsymbol{w}(1, t) \times \boldsymbol{D}(1, t)]\right\} \cdot \mathbf{c} .
\end{aligned}
$$

Constitutive equations. To simplify many expressions involving the constitutive equations we set

$$
\boldsymbol{\xi}:=(\mathbf{v}, \mathbf{u})
$$


The rod is assumed to be viscoelastic of strain-rate type, so that there are continuously differentiable constitutive functions

$$
(\boldsymbol{\xi}, \dot{\boldsymbol{\xi}}, s) \mapsto \hat{\mathbf{n}}(\boldsymbol{\xi}, \dot{\boldsymbol{\xi}}, s), \hat{\mathbf{m}}(\boldsymbol{\xi}, \dot{\boldsymbol{\xi}}, s), \varphi(\boldsymbol{\xi}, s), \mathbf{n}_{\mathrm{D}}(\boldsymbol{\xi}, \dot{\boldsymbol{\xi}}, s), \mathbf{m}_{\mathrm{D}}(\boldsymbol{\xi}, \dot{\boldsymbol{\xi}}, s)
$$

such that the rod has constitutive equations (invariant under rigid motions) of the form

$$
\begin{aligned}
\mathbf{n}(s, t) & =\hat{\mathbf{n}}\left(\boldsymbol{\xi}(s, t), \boldsymbol{\xi}_{t}(s, t), s\right):=\varphi_{\mathbf{v}}(\boldsymbol{\xi}(s, t), s)+\mathbf{n}_{\mathrm{D}}\left(\boldsymbol{\xi}(s, t), \boldsymbol{\xi}_{t}(s, t), s\right), \\
\mathbf{m}(s, t) & =\hat{\mathbf{m}}\left(\boldsymbol{\xi}(s, t), \boldsymbol{\xi}_{t}(s, t), s\right):=\varphi_{\mathbf{u}}(\boldsymbol{\xi}(s, t), s)+\mathbf{m}_{\mathrm{D}}\left(\boldsymbol{\xi}(s, t), \boldsymbol{\xi}_{t}(s, t), s\right)
\end{aligned}
$$

where $\mathbf{n}:=\left(\boldsymbol{n} \cdot \boldsymbol{d}_{1}, \boldsymbol{n} \cdot \boldsymbol{d}_{2}, \boldsymbol{n} \cdot \boldsymbol{d}_{3}\right) \equiv \boldsymbol{n} \cdot \mathbf{D}$, etc., and where

$$
\mathbf{n}_{\mathrm{D}}(\mathbf{v}, \mathbf{u}, \mathbf{o}, \mathbf{o}, s)=\mathbf{o}, \quad \mathbf{m}_{\mathrm{D}}(\mathbf{v}, \mathbf{u}, \mathbf{o}, \mathbf{o}, s)=\mathbf{o} .
$$

The superposed dot on $\boldsymbol{\xi}$ has no operational significance: It merely identifies an argument whose slot is to be occupied by a time derivative. The domains of these functions are defined by (2.13). $\varphi$ is the stored-energy function accounting for the equilibrium response in light of (2.31). We assume that there is a constant $c>0$ such that

$$
\begin{aligned}
& \mathbf{a} \cdot \frac{\partial \hat{\mathbf{n}}}{\partial \dot{\mathbf{v}}} \cdot \mathbf{a}+\mathbf{a} \cdot \frac{\partial \hat{\mathbf{n}}}{\partial \dot{\mathbf{u}}} \cdot \mathbf{b}+\mathbf{b} \cdot \frac{\partial \hat{\mathbf{m}}}{\partial \dot{\mathbf{v}}} \cdot \mathbf{a}+\mathbf{b} \cdot \frac{\partial \hat{\mathbf{m}}}{\partial \dot{\mathbf{u}}} \cdot \mathbf{b} \\
\equiv & \mathbf{a} \cdot \frac{\partial \hat{\mathbf{n}}_{\mathrm{D}}}{\partial \dot{\mathbf{v}}} \cdot \mathbf{a}+\mathbf{a} \cdot \frac{\partial \hat{\mathbf{n}}_{\mathrm{D}}}{\partial \dot{\mathbf{u}}} \cdot \mathbf{b}+\mathbf{b} \cdot \frac{\partial \hat{\mathbf{m}}_{\mathrm{D}}}{\partial \dot{\mathbf{v}}} \cdot \mathbf{a}+\mathbf{b} \cdot \frac{\partial \hat{\mathbf{m}}_{\mathrm{D}}}{\partial \dot{\mathbf{u}}} \cdot \mathbf{b} \geq c\left(|\mathbf{a}|^{2}+|\mathbf{b}|^{2}\right) \quad \forall \mathbf{a}, \mathbf{b} \in \mathbb{R}^{3} .
\end{aligned}
$$

This uniform monotonicity condition ensures that the response is truly dissipative and that the governing equations of motion have a parabolic character. In view of the smoothness of these constitutive functions, (2.32) is equivalent to

$$
\begin{aligned}
& {\left[\hat{\mathbf{n}}\left(\boldsymbol{\xi}, \dot{\boldsymbol{\xi}}_{2}, s\right)-\hat{\mathbf{n}}\left(\boldsymbol{\xi}, \dot{\boldsymbol{\xi}}_{1}, s\right)\right] \cdot\left[\dot{\mathbf{v}}_{2}-\dot{\mathbf{v}}_{1}\right]+\left[\hat{\mathbf{m}}\left(\boldsymbol{\xi}, \dot{\boldsymbol{\xi}}_{2}, s\right)-\hat{\mathbf{m}}\left(\boldsymbol{\xi}, \dot{\boldsymbol{\xi}}_{1}, s\right)\right] \cdot\left[\dot{\mathbf{u}}_{2}-\dot{\mathbf{u}}_{1}\right]} \\
& \quad \geq c\left\{\left|\dot{\mathbf{v}}_{2}-\dot{\mathbf{v}}_{1}\right|^{2}+\left|\dot{\mathbf{u}}_{2}-\dot{\mathbf{u}}_{1}\right|^{2}\right\} \equiv c\left|\dot{\boldsymbol{\xi}}_{2}-\dot{\boldsymbol{\xi}}_{1}\right|^{2}
\end{aligned}
$$

which in turn is equivalent to the inequality in which $(\hat{\mathbf{n}}, \hat{\mathbf{m}})$ is replaced with $\left(\mathbf{n}_{\mathrm{D}}, \mathbf{m}_{\mathrm{D}}\right)$.

Conditions (2.32) and (2.33) support a global implicit-function theorem (based on degree theory) that asserts that the system of finite-dimensional equations

$$
\hat{\mathbf{n}}(\boldsymbol{\xi}, \dot{\boldsymbol{\xi}}, s)=\mathbf{n}, \quad \hat{\mathbf{m}}(\boldsymbol{\xi}, \dot{\boldsymbol{\xi}}, s)=\mathbf{m}
$$

can be solved uniquely for $\dot{\boldsymbol{\xi}}$ in terms of the other variables:

$$
\dot{\boldsymbol{\xi}}=\dot{\boldsymbol{\xi}}^{\sharp}(\boldsymbol{\xi}, \mathbf{n}, \mathbf{m}, s)
$$

with $\dot{\boldsymbol{\xi}}^{\sharp}$ inheriting the regularity of $\hat{\mathbf{n}}$ and $\hat{\mathbf{m}}$ and satisfying the monotonicity condition:

$$
\frac{\partial\left(\dot{\mathbf{v}}^{\sharp}, \dot{\mathbf{u}}^{\sharp}\right)}{\partial(\mathbf{n}, \mathbf{m})} \quad \text { is positive-definite. }
$$

Thus (2.30) is equivalent to

$$
\boldsymbol{\xi}_{t}(s, t)=\dot{\boldsymbol{\xi}}^{\sharp}(\boldsymbol{\xi}, \mathbf{n}(s, t), \mathbf{m}(s, t), s) .
$$

Equations first-order in time. To convert our system of equations into one with only first-order time derivatives, let

$$
\boldsymbol{p}=\boldsymbol{r}_{t}, \quad \boldsymbol{v}:=\boldsymbol{r}_{s},
$$


so that our system is equivalent to

$$
\begin{gathered}
\varepsilon \rho A \boldsymbol{p}_{t}=\boldsymbol{n}_{s}, \\
\varepsilon[(\rho \boldsymbol{I})(s, t) \cdot \boldsymbol{w}(s, t)]_{t}=\boldsymbol{m}_{s}+\boldsymbol{v} \times \boldsymbol{n}, \\
\boldsymbol{v}_{t}=\boldsymbol{p}_{s} \Longleftrightarrow \mathbf{v}_{t}+\mathbf{w} \times \mathbf{v}=\mathbf{p}_{s}+\mathbf{u} \times \mathbf{p} \Longleftrightarrow \mathbf{v}_{t}=\mathbf{D}^{\top} \cdot \boldsymbol{p}_{s}-\mathbf{w} \times \mathbf{v}, \\
\boldsymbol{u}_{t}=\boldsymbol{w}_{s}+\boldsymbol{w} \times \boldsymbol{u} \quad \Longleftrightarrow \quad \mathbf{u}_{t}=\mathbf{w}_{s}+\mathbf{u} \times \mathbf{w} \equiv \mathbf{D}^{\top} \cdot \boldsymbol{w}_{s}, \\
\mu\left\{\boldsymbol{p}_{t}(1, t)+\boldsymbol{w}_{t}(1, t) \times \boldsymbol{c}(t)+\boldsymbol{w}(1, t) \times[\boldsymbol{w}(1, t) \times \boldsymbol{c}(t)]\right\}=-\boldsymbol{n}(1, t), \\
{[\boldsymbol{J}(t) \cdot \boldsymbol{w}(1, t)]_{t}=-\boldsymbol{m}(1, t)+\boldsymbol{c}(t) \times \boldsymbol{n}(1, t) .}
\end{gathered}
$$

Note the distinctions between the equations in (2.42). We adopt general initial conditions

$$
\boldsymbol{r}(s, 0)=\boldsymbol{r}^{\circ}(s), \boldsymbol{D}(s, 0)=\boldsymbol{D}^{\circ}(s), \boldsymbol{r}_{t}(s, 0)=\boldsymbol{p}^{\circ}(s), \boldsymbol{w}(s, 0)=\boldsymbol{w}^{\circ}(s) \text { for } s \in[0,1] .
$$

Compatibility of initial and boundary conditions. To effect the asymptotic analysis, we require the solutions to have a level of smoothness corresponding to the order of the perturbation. For this purpose we require that the initial data be sufficiently regular and satisfy compatibility conditions of higher order at $(s, t)=(0,0),(1,0)$. That (2.14) implies that $\partial_{t}^{k} \boldsymbol{p}(0, t)=\boldsymbol{o}=\partial_{t}^{k} \boldsymbol{w}(0, t)$ for $k=1,2, \ldots$ leads to the compatibility equations at $(0,0)$, with those equations of higher order obtained by exploiting the equations of motion:

$$
\begin{gathered}
\boldsymbol{r}^{\circ}(0)=\boldsymbol{o}, \quad \boldsymbol{D}^{\circ}(0)=\boldsymbol{i}_{k} \mathbf{e}_{k}, \quad \boldsymbol{p}^{\circ}(0)=\boldsymbol{o}, \quad \boldsymbol{w}^{\circ}(0)=\boldsymbol{o}, \\
\partial_{s}\left[\boldsymbol { D } ^ { \circ } ( s ) \cdot \hat { \mathbf { n } } \left(\boldsymbol{\xi}(s, 0), \mathbf{p}_{s}^{\circ}(s)+\mathbf{u}(s, 0) \times \mathbf{p}^{\circ}(s)-\mathbf{w}^{\circ}(s) \times \mathbf{v}(s, 0),\right.\right. \\
\left.\left.\mathbf{w}_{s}^{\circ}(s)+\mathbf{u}(s, 0) \times \mathbf{w}^{\circ}(s)\right)\right]\left.\right|_{s=0}=\boldsymbol{o},
\end{gathered}
$$

etc., where

$$
\boldsymbol{D}^{\circ}(s) \cdot \mathbf{v}(s, 0)=\boldsymbol{r}_{s}^{\circ}(s), \quad \boldsymbol{D}_{s}^{\circ}(s)=\mathbf{u}(s, 0) \times \boldsymbol{D}^{\circ}(s) .
$$

To get a first-order compatibility condition at $(s, t)=(1,0)$ we eliminate $\boldsymbol{p}_{t}(1, t)$ between (2.39) and (2.43):

$$
\left.\boldsymbol{n}_{s}\right|_{\substack{s=1 \\ t=0}}=\varepsilon \rho A\left\{-\left.\mu^{-1} \boldsymbol{n}\right|_{\substack{s=1 \\ t=0}}-\boldsymbol{w}_{t}(1,0) \times \boldsymbol{D}^{\circ}(1) \cdot \mathbf{c}+\boldsymbol{w}^{\circ}(1) \times\left[\boldsymbol{w}^{\circ}(1) \times \boldsymbol{D}^{\circ}(1) \cdot \mathbf{c}\right]\right\}
$$

where $\boldsymbol{w}_{t}(1,0)$ is found from an analogous treatment of (2.44), which we do not pause to exhibit. Higher-order compatibility conditions are found similarly.

Stretched equations. We make the change of variables

$$
t=\varepsilon \tau,
$$

and for any function $(s, t) \mapsto \psi(s, t)$ we set

$$
\psi^{\star}(s, \tau):=\psi(s, \varepsilon \tau) .
$$


The substitution of (2.50) into the system (2.39)-(2.44) puts them into their stretched form

$$
\begin{gathered}
\rho A \boldsymbol{p}_{\tau}^{\star} \equiv \rho A\left[\varepsilon\left(\boldsymbol{w}^{\star} \times \boldsymbol{D}^{\star}\right) \cdot \mathbf{p}^{\star}+\boldsymbol{D}^{\star} \cdot \mathbf{p}_{\tau}^{\star}\right]=\boldsymbol{n}_{s}^{\star}, \\
{\left[(\rho \boldsymbol{I})^{\star}(s, \tau) \cdot \boldsymbol{w}^{\star}(s, \tau)\right]_{\tau} \equiv\left[\boldsymbol{D}^{\star} \cdot \rho \mathbf{I} \cdot \mathbf{D}^{\star \top} \cdot \boldsymbol{w}^{\star}\right]_{\tau} \equiv\left[\boldsymbol{D}^{\star} \cdot \rho \mathbf{I} \cdot \mathbf{w}^{\star}\right]_{\tau}} \\
=\boldsymbol{m}_{s}^{\star}+\boldsymbol{v}^{\star} \times \boldsymbol{n}^{\star}, \\
\varepsilon^{-1} \boldsymbol{v}_{\tau}^{\star}=\boldsymbol{p}_{s}^{\star} \Longleftrightarrow \varepsilon^{-1} \mathbf{v}_{\tau}^{\star}+\mathbf{w}^{\star} \times \mathbf{v}^{\star}=\mathbf{p}_{s}^{\star}+\mathbf{u}^{\star} \times \mathbf{p}^{\star}, \\
\varepsilon^{-1} \boldsymbol{u}_{\tau}^{\star}=\boldsymbol{w}_{s}^{\star}+\boldsymbol{w}^{\star} \times \boldsymbol{u}^{\star} \quad \Longleftrightarrow \quad \varepsilon^{-1} \mathbf{u}_{\tau}^{\star}=\mathbf{w}_{s}^{\star}+\mathbf{u}^{\star} \times \mathbf{w}^{\star}, \\
\mu\left\{\boldsymbol{p}_{\tau}^{\star}(1, \tau)+\boldsymbol{w}_{\tau}^{\star}(1, \tau) \times \boldsymbol{c}^{\star}(\tau)+\varepsilon \boldsymbol{w}^{\star}(1, \tau) \times\left[\boldsymbol{w}^{\star}(1, \tau) \times \boldsymbol{c}^{\star}(\tau)\right]\right\}=-\varepsilon \boldsymbol{n}^{\star}(1, t), \\
{\left[\boldsymbol{J}^{\star}(\tau) \cdot \boldsymbol{w}^{\star}(1, \tau)\right]_{\tau} \equiv\left[\boldsymbol{D}^{\star}(1, \tau) \cdot \mathbf{J} \cdot \mathbf{w}(1, \tau)\right]_{\tau}=-\varepsilon \boldsymbol{m}^{\star}(1, \tau)+\varepsilon \boldsymbol{c}^{\star}(\tau) \times \boldsymbol{n}^{\star}(1, \tau)}
\end{gathered}
$$

where

$$
\boldsymbol{n}^{\star}(s, \tau)=\boldsymbol{D}^{\star}(s, \tau) \cdot \hat{\mathbf{n}}\left(\boldsymbol{\xi}^{\star}(s, \tau), \varepsilon^{-1} \boldsymbol{\xi}_{\tau}^{\star}(s, \tau), s\right), \quad \text { etc. }
$$

Existence and regularity. Throughout this paper we make the blanket regularity assumption that all data $\hat{\mathbf{n}}, \hat{\mathbf{m}}, \boldsymbol{r}^{\circ}, \boldsymbol{D}^{\circ}, \boldsymbol{p}^{\circ}, \boldsymbol{w}^{\circ}, \rho A, \rho \mathbf{I}$ have as many continuous derivatives as are needed in the analysis. Likewise, we assume that the initial data satisfy corner compatibility conditions of whatever order is needed. The basic (global) existence theory for this problem is given in [4]. Some of the techniques used in this reference are similar to those in Section 8 .

\section{Asymptotic expansions. Let}

$$
\boldsymbol{\phi}:=(\boldsymbol{r}, \boldsymbol{D}, \boldsymbol{v}, \boldsymbol{u}, \mathbf{v}, \mathbf{u}), \quad \boldsymbol{\psi}:=(\boldsymbol{p}, \boldsymbol{w}, \mathbf{p}, \mathbf{w}) .
$$

$\boldsymbol{\phi}$ determines the configuration and $\boldsymbol{\psi}$ determines the velocity. We seek an asymptotic representation for solutions of the governing equations in the form

$$
\left\{\begin{array}{c}
\boldsymbol{\phi}(s, t ; \varepsilon) \\
\boldsymbol{\psi}(s, t ; \varepsilon)
\end{array}\right\}=\left\{\begin{array}{l}
\boldsymbol{\phi}_{\mathrm{A}}^{H}(s, t ; \varepsilon)+o\left(\varepsilon^{H}\right) \\
\boldsymbol{\psi}_{\mathrm{A}}^{H}(s, t ; \varepsilon)+o\left(\varepsilon^{H}\right)
\end{array}\right\},\left\{\begin{array}{l}
\boldsymbol{\phi}_{\mathrm{A}}^{H}(s, t ; \varepsilon) \\
\boldsymbol{\psi}_{\mathrm{A}}^{H}(s, t ; \varepsilon)
\end{array}\right\}:=\left\{\begin{array}{c}
\boldsymbol{\phi}_{\mathrm{R}}^{H}(s, t ; \varepsilon)+\boldsymbol{\phi}_{\mathrm{L}}^{H}(s, t / \varepsilon ; \varepsilon) \\
\boldsymbol{\psi}_{\mathrm{R}}^{H}(s, t ; \varepsilon)+\boldsymbol{\psi}_{\mathrm{L}}^{H}(s, t / \varepsilon ; \varepsilon)
\end{array}\right\}
$$

where

$$
\begin{gathered}
\left\{\begin{array}{c}
\boldsymbol{\phi}_{\mathrm{R}}^{H}(s, t ; \varepsilon) \\
\boldsymbol{\psi}_{\mathrm{R}}^{H}(s, t ; \varepsilon)
\end{array}\right\}:=\sum_{j=0}^{H}\left\{\begin{array}{l}
\boldsymbol{\phi}_{j}(s, t) \\
\boldsymbol{\psi}_{j}(s, t)
\end{array}\right\} \frac{\varepsilon^{j}}{j !}, \\
\boldsymbol{\phi}_{\mathrm{L}}^{H}(s, \tau ; \varepsilon):=\sum_{j=0}^{H-1} \boldsymbol{\phi}_{j}^{\star}(s, \tau) \frac{\varepsilon^{j+1}}{(j+1) !}, \quad \boldsymbol{\psi}_{\mathrm{L}}^{H}(s, \tau ; \varepsilon):=\sum_{j=0}^{H} \boldsymbol{\psi}_{j}^{\star}(s, \tau) \frac{\varepsilon^{j}}{j !}
\end{gathered}
$$

with

$$
\partial_{s}^{p} \partial_{t}^{q} \boldsymbol{\phi}_{j}^{\star}(s, \tau), \partial_{s}^{p} \partial_{t}^{q} \boldsymbol{\psi}_{j}^{\star}(s, \tau) \rightarrow 0 \quad \text { as } \quad \tau \rightarrow \infty
$$

for all the derivatives $\partial_{s}^{p} \partial_{t}^{q}$ that appear in our analysis. The error term $o\left(\varepsilon^{H}\right)$ is measured in a suitable norm for $\boldsymbol{\phi}$ and $\boldsymbol{\psi}$ and all such derivatives. $\left(\boldsymbol{\phi}_{\mathrm{A}}^{H}, \boldsymbol{\psi}_{\mathrm{A}}^{H}\right)$ is the asymptotic expansion (to order $H),\left(\boldsymbol{\phi}_{\mathrm{R}}^{H}, \boldsymbol{\psi}_{\mathrm{R}}^{H}\right)$ is the regular expansion, and $\left(\boldsymbol{\phi}_{\mathrm{L}}^{H}, \boldsymbol{\psi}_{\mathrm{L}}^{H}\right)$ is the initiallayer expansion. The difference in the dispositions of $\varepsilon$ in the initial-layer expansions (3.4) (which is not essential) simplifies the asymptotic formulas to follow. 
We get the equations for the terms of order $l$ of the regular expansion by substituting the regular expansion into all the governing equations of Section 2 except the second two initial conditions of (2.45), differentiating these equations $l$ times with respect to $\varepsilon$, and setting $\varepsilon=0$. For this purpose we note that

$$
\left\{\begin{array}{l}
\boldsymbol{\phi}_{j}(s, t) \\
\boldsymbol{\psi}_{j}(s, t)
\end{array}\right\}=\left\{\begin{array}{c}
\partial_{\varepsilon}^{j} \boldsymbol{\phi}_{\mathrm{R}}^{H}(s, t ; 0) \\
\partial_{\varepsilon}^{j} \boldsymbol{\psi}_{\mathrm{R}}^{H}(s, t ; 0)
\end{array}\right\}, \quad j=0, \ldots, k .
$$

For treating the terms of the initial-layer expansion, we shall likewise treat the stretched equations (2.51)-(2.56) together with the following versions of (3.2)-(3.4):

$$
\begin{aligned}
\boldsymbol{\phi}_{\mathrm{A}}^{H}(s, \varepsilon \tau ; \varepsilon) & =\sum_{j=0}^{H} \boldsymbol{\phi}_{j}(s, \varepsilon \tau) \frac{\varepsilon^{j}}{j !}+\sum_{j=0}^{H-1} \boldsymbol{\phi}_{j}^{\star}(s, \tau) \frac{\varepsilon^{j+1}}{(j+1) !}, \\
\partial_{\tau} \boldsymbol{\phi}_{\mathrm{A}}^{H}(s, \varepsilon \tau ; \varepsilon)= & \sum_{j=0}^{H} \boldsymbol{\phi}_{j t}(s, \varepsilon \tau) \frac{\varepsilon^{j+1}}{j !}+\sum_{j=0}^{H-1} \boldsymbol{\phi}_{j \tau}^{\star}(s, \tau) \frac{\varepsilon^{j+1}}{(j+1) !}, \\
\partial_{\varepsilon} \boldsymbol{\phi}_{\mathrm{A}}^{H}(s, \varepsilon \tau ; \varepsilon)= & \sum_{j=0}^{H} \tau \boldsymbol{\phi}_{j t}(s, \varepsilon \tau) \frac{\varepsilon^{j}}{j !}+\sum_{j=1}^{H} \boldsymbol{\phi}_{j}(s, \varepsilon \tau) \frac{\varepsilon^{j-1}}{(j-1) !}+\sum_{j=0}^{H-1} \boldsymbol{\phi}_{j}^{\star}(s, \tau) \frac{\varepsilon^{j}}{j !}, \\
\partial_{\varepsilon \tau} \boldsymbol{\phi}_{\mathrm{A}}^{H}(s, \varepsilon \tau ; \varepsilon)= & \sum_{j=0}^{H} \tau \boldsymbol{\phi}_{j t t}(s, \varepsilon \tau) \frac{\varepsilon^{j+1}}{j !}+\sum_{j=0}^{H}(j+1) \boldsymbol{\phi}_{j t}(s, \varepsilon \tau) \frac{\varepsilon^{j}}{j !}+\sum_{j=0}^{H-1} \boldsymbol{\phi}_{j \tau}^{\star}(s, \tau) \frac{\varepsilon^{j}}{j !},
\end{aligned}
$$

so that

$$
\begin{gathered}
\boldsymbol{\phi}_{\mathrm{A}}^{H}(s, 0 ; 0)=\boldsymbol{\phi}_{0}(s, 0), \\
\left.\partial_{\tau} \boldsymbol{\phi}_{\mathrm{A}}^{H}(s, 0 ; \varepsilon)\right]=\mathbf{o}, \\
\lim _{\varepsilon \rightarrow 0}\left[\varepsilon^{-1} \partial_{\tau} \boldsymbol{\phi}_{\mathrm{A}}^{H}(s, \varepsilon \tau ; \varepsilon)\right]=\boldsymbol{\phi}_{0 t}(s, 0)+\boldsymbol{\phi}_{0 \tau}^{\star}(s, \tau), \\
\partial_{\varepsilon} \boldsymbol{\phi}_{\mathrm{A}}^{H}(s, 0 ; 0)=\tau \boldsymbol{\phi}_{0 t}(s, 0)+\boldsymbol{\phi}_{1}(s, 0)+\boldsymbol{\phi}_{0}^{\star}(s, \tau), \\
\partial_{\varepsilon \tau} \boldsymbol{\phi}_{\mathrm{A}}^{H}(s, 0 ; 0)=\boldsymbol{\phi}_{0 t}(s, 0)+\boldsymbol{\phi}_{0 \tau}^{\star}(s, \tau), \\
\partial_{\varepsilon \varepsilon \tau} \boldsymbol{\phi}_{\mathrm{A}}^{H}(s, 0 ; 0)=2 \tau \boldsymbol{\phi}_{0 t t}(s, 0)+2 \boldsymbol{\phi}_{1 t}(s, 0)+\boldsymbol{\phi}_{1 \tau}^{\star}(s, \tau), \\
\boldsymbol{\psi}_{\mathrm{A}}^{H}(s, \varepsilon \tau ; \varepsilon)=\sum_{j=0}^{H} \boldsymbol{\psi}_{j}(s, \varepsilon \tau) \frac{\varepsilon^{j}}{j !}+\sum_{j=0}^{H} \boldsymbol{\psi}_{j}^{\star}(s, \tau) \frac{\varepsilon^{j}}{j !}, \\
\partial_{\tau} \boldsymbol{\psi}_{\mathrm{A}}^{H}(s, \varepsilon \tau ; \varepsilon)=\sum_{j=0}^{H} \boldsymbol{\psi}_{j t}(s, \varepsilon \tau) \frac{\varepsilon^{j+1}}{j !}+\sum_{j=0}^{H} \boldsymbol{\psi}_{j \tau}^{\star}(s, \tau) \frac{\varepsilon^{j}}{j !}, \\
\partial_{\varepsilon} \boldsymbol{\psi}_{\mathrm{A}}^{H}(s, \varepsilon \tau ; \varepsilon)=\sum_{j=0}^{H} \tau \boldsymbol{\psi}_{j t}(s, \varepsilon \tau) \frac{\varepsilon^{j}}{j !}+\sum_{j=1}^{H} \boldsymbol{\psi}_{j}(s, \varepsilon \tau) \frac{\varepsilon^{j-1}}{(j-1) !}+\sum_{j=1}^{H} \boldsymbol{\psi}_{j}^{\star}(s, \tau) \frac{\varepsilon^{j-1}}{(j-1) !}, \\
\partial_{\varepsilon \tau} \boldsymbol{\psi}_{\mathrm{A}}^{H}(s, \varepsilon \tau ; \varepsilon)=\sum_{j=0}^{H} \tau \boldsymbol{\psi}_{j t t}(s, \varepsilon \tau) \frac{\varepsilon^{j+1}}{j !}+\sum_{j=0}^{H}(j+1) \boldsymbol{\psi}_{j t}(s, \varepsilon \tau) \frac{\varepsilon^{j}}{j !}+\sum_{j=1}^{H} \boldsymbol{\psi}_{j \tau}^{\star}(s, \tau) \frac{\varepsilon^{j-1}}{(j-1) !},
\end{gathered}
$$


so that

$$
\begin{aligned}
\boldsymbol{\psi}_{\mathrm{A}}^{H}(s, 0 ; 0) & =\boldsymbol{\psi}_{0}(s, 0)+\boldsymbol{\psi}_{0}^{\star}(s, \tau), \\
\partial_{\tau} \boldsymbol{\psi}_{\mathrm{A}}^{H}(s, 0 ; 0) & =\boldsymbol{\psi}_{0 \tau}^{\star}(s, \tau), \\
\partial_{\varepsilon} \boldsymbol{\psi}_{\mathrm{A}}^{H}(s, 0 ; 0) & =\tau \boldsymbol{\psi}_{0 t}(s, 0)+\boldsymbol{\psi}_{1}(s, 0)+\boldsymbol{\psi}_{1}^{\star}(s, \tau), \\
\partial_{\varepsilon \tau} \boldsymbol{\psi}_{\mathrm{A}}^{H}(s, 0 ; 0) & =\boldsymbol{\psi}_{0 t}(s, 0)+\boldsymbol{\psi}_{1 \tau}^{\star}(s, \tau) .
\end{aligned}
$$

It would be consistent with our notational scheme but too cumbersome to have the left-hand sides of (3.7)-(3.10) carry stars.

4. The reduced problem. Substituting the regular expansion (3.3) into (2.21)(2.24), (2.37), (2.14), (2.45) and setting $\varepsilon=0$ yields the reduced problem, which governs the leading terms of the regular expansion:

$$
\begin{gathered}
\boldsymbol{o}=\boldsymbol{n}_{0 s} \equiv\left(\boldsymbol{D}_{0} \cdot \mathbf{n}_{0}\right)_{s}, \\
\boldsymbol{o}=\boldsymbol{m}_{0 s}+\boldsymbol{v}_{0} \times \boldsymbol{n}_{0} \equiv\left(\boldsymbol{D}_{0} \cdot \mathbf{m}_{0}\right)_{s}+\boldsymbol{v}_{0} \times \boldsymbol{n}_{0}, \\
\mu\left\{\boldsymbol{r}_{0 t t}(1, t)+\boldsymbol{w}_{0 t}(1, t) \times \boldsymbol{c}_{0}(t)+\boldsymbol{w}_{0}(1, t) \times\left[\boldsymbol{w}_{0}(1, t) \times \boldsymbol{c}_{0}(t)\right]\right\}=-\boldsymbol{n}_{0}(1, t), \\
{\left[\boldsymbol{J}_{0}(t) \cdot \boldsymbol{w}_{0}(1, t)\right]_{t}=-\boldsymbol{m}_{0}(1, t)+\boldsymbol{c}_{0}(t) \times \boldsymbol{n}_{0}(1, t),} \\
\boldsymbol{D}_{0 t}=\boldsymbol{w}_{0} \times \boldsymbol{D}_{0}, \\
\boldsymbol{\xi}_{0 t}(s, t)=\dot{\boldsymbol{\xi}}^{\sharp}\left(\boldsymbol{\xi}_{0}(s, t), \mathbf{n}_{0}(s, t), \mathbf{m}_{0}(s, t), s\right), \\
\boldsymbol{r}_{0}(0, t)=0, \quad \boldsymbol{D}_{0}(0, t)=\boldsymbol{i}_{k} \mathbf{e}_{k}, \\
\boldsymbol{r}_{0}(s, 0)=\boldsymbol{r}^{\circ}(s), \quad \boldsymbol{D}_{0}(s, 0)=\boldsymbol{D}^{\circ}(s), \\
\boldsymbol{r}_{0 t}(1,0)=\boldsymbol{p}^{\circ}(1), \quad \boldsymbol{w}_{0}(1,0)=\boldsymbol{w}^{\circ}(1)
\end{gathered}
$$

where

$$
\begin{aligned}
\mathbf{n}_{0}(s, t) & :=\boldsymbol{n}_{0}(1, t) \cdot \boldsymbol{D}_{0}(s, t), \\
\mathbf{m}_{0}(s, t) & :=\left\{\boldsymbol{m}_{0}(1, t)+\boldsymbol{q}(s, t) \times \boldsymbol{n}_{0}(1, t)\right\} \cdot \boldsymbol{D}_{0}(s, t), \\
\boldsymbol{q}(s, t) & :=\boldsymbol{r}_{0}(1, t)-\boldsymbol{r}_{0}(s, t) .
\end{aligned}
$$

The initial conditions (4.8) and (4.9), as we shall show, are adequate for the unique solvability of the reduced problem. Condition (4.8) implies that

$$
\boldsymbol{v}_{0}(s, 0)=\boldsymbol{v}^{\circ}(s), \quad \boldsymbol{u}_{0}(s, 0)=\boldsymbol{u}^{\circ}(s) .
$$

Initial conditions coming from (2.45) ${ }_{3,4}$ are coupled with those of the initial-layer expansion, and are therefore not exhibited until Section 6 .

We sketch the treatment of the reduced problem from [3]: Since (2.6)-(2.12) imply that

$$
\boldsymbol{r}_{0 s t}=\boldsymbol{D}_{0} \cdot \mathbf{v}_{0 t}+\boldsymbol{w}_{0} \times \boldsymbol{r}_{0 s}, \quad \boldsymbol{w}_{0 s}=\boldsymbol{D}_{0} \cdot \mathbf{u}_{0 t},
$$


an integration by parts yields

$$
\begin{aligned}
\boldsymbol{r}_{0 t}(s, t) & =\int_{0}^{s} \boldsymbol{D}_{0}(\xi, t) \cdot \mathbf{v}_{0 t}(\xi, t)-\left[\boldsymbol{r}_{0}(s, t)-\boldsymbol{r}_{0}(\xi, t)\right] \times \boldsymbol{D}_{0}(\xi, t) \cdot \mathbf{u}_{0 t}(\xi, t) d \xi, \\
\boldsymbol{w}_{0}(s, t) & =\int_{0}^{s} \boldsymbol{D}_{0}(\xi, t) \cdot \mathbf{u}_{0 t}(\xi, t) d \xi \\
\boldsymbol{D}_{0 t}(s, t) & =\boldsymbol{w}_{0}(s, t) \times \boldsymbol{D}_{0}(s, t)
\end{aligned}
$$

where $\mathbf{v}_{0 t}$ and $\mathbf{u}_{0 t}$ are given by (4.6).

If $\boldsymbol{m}_{0}(1, \cdot)$ and $\boldsymbol{n}_{0}(1, \cdot)$ were known, then (4.3)-(4.6), together with (4.13)-4.15), would constitute a system of ordinary differential equations for the family

$$
\left\{\mathbf{v}_{0}(s, \cdot), \mathbf{u}_{0}(s, \cdot), \boldsymbol{r}_{0}(s, \cdot), \boldsymbol{D}_{0}(s, \cdot), \boldsymbol{w}_{0}(s, \cdot): s \in[0,1]\right\} .
$$

That this family of functions is parametrized by $s$ is just the remnant of the full partial differential equations valid for $\varepsilon \neq 0$. We now show how to augment this system with ordinary differential equations for $\boldsymbol{m}(1, \cdot)$ and $\boldsymbol{n}(1, \cdot)$.

We first solve (4.4) for $\boldsymbol{w}_{0 t}$, substitute the result into (4.3), then compute $\boldsymbol{r}_{0 t t}$ from (4.13), and also substitute it into (4.3) to obtain

$$
\begin{aligned}
& -\mu^{-1} \boldsymbol{n}_{0}(1, t)+\cdots=\boldsymbol{r}_{0 t t}(1, t) \\
& \equiv \int_{0}^{1}\left\{\boldsymbol{D}_{0}(\xi, t) \cdot \mathbf{v}_{0 t t}(\xi, t)-\boldsymbol{q}(\xi, t) \times \boldsymbol{D}_{0}(\xi, t) \cdot \mathbf{u}_{0 t t}(\xi, t)\right\} d \xi+\cdots \\
& \equiv \int_{0}^{1}\left\{\boldsymbol{D}_{0} \cdot\left[\dot{\mathbf{v}}_{\mathbf{n}}^{\sharp} \cdot \mathbf{n}_{0 t}(\xi, t)+\dot{\mathbf{v}}_{\mathbf{m}}^{\sharp} \cdot \mathbf{m}_{0 t}(\xi, t)\right]\right. \\
& \left.\quad-\boldsymbol{q}(\xi, t) \times \boldsymbol{D}_{0}(\xi, t) \cdot\left[\dot{\mathbf{u}}_{\mathbf{n}}^{\sharp} \cdot \mathbf{n}_{0 t}(\xi, t)+\dot{\mathbf{u}}_{\mathbf{m}}^{\sharp} \cdot \mathbf{m}_{0 t}(\xi, t)\right]\right\} d \xi+\cdots \\
& \equiv \int_{0}^{1}\left\{\boldsymbol{D}_{0} \cdot\left[\dot{\mathbf{v}}_{\mathbf{n}}^{\sharp} \cdot \mathbf{D}_{0}^{\top} \cdot \boldsymbol{n}_{0 t}(1, t)+\dot{\mathbf{v}}_{\mathbf{m}}^{\sharp} \cdot \mathbf{D}_{0}^{\top} \cdot\left(\boldsymbol{m}_{0 t}(1, t)+\boldsymbol{q} \times \boldsymbol{n}_{0 t}(1,0)\right)\right]\right. \\
& \left.\quad \quad-\boldsymbol{q} \times \boldsymbol{D}_{0} \cdot\left[\dot{\mathbf{u}}_{\mathbf{n}}^{\sharp} \cdot \mathbf{D}_{0}^{\top} \cdot \boldsymbol{n}_{0 t}(1, t)+\dot{\mathbf{u}}_{\mathbf{m}}^{\sharp} \cdot \mathbf{D}_{0}^{\top} \cdot\left(\boldsymbol{m}_{0 t}(1, t)+\boldsymbol{q} \times \boldsymbol{n}_{0 t}(1,0)\right)\right]\right\} d \xi+\cdots
\end{aligned}
$$

where the ellipses stand for terms that do not contain $\boldsymbol{n}_{0 t}(1, t)$ and $\boldsymbol{m}_{0 t}(1, t)$, their meaning changing in each line. Likewise,

$$
\begin{aligned}
& -\boldsymbol{J}_{0}(t)^{-1} \cdot \boldsymbol{m}_{0}(1, t)+\cdots=\boldsymbol{w}_{0 t}(1, t) \\
& \equiv \int_{0}^{1} \boldsymbol{D}_{0} \cdot\left[\dot{\mathbf{u}}_{\mathbf{n}}^{\sharp} \cdot \mathbf{D}_{0}^{\top} \cdot \boldsymbol{n}_{0 t}(1, t)+\dot{\mathbf{u}}_{\mathbf{m}}^{\sharp} \cdot \mathbf{D}_{0}^{\top} \cdot\left(\mathbf{m}_{0 t}(1, t)+\boldsymbol{q} \times \boldsymbol{n}_{0 t}(1,0)\right)\right] d \xi+\cdots
\end{aligned}
$$

By using the identities

$$
\begin{aligned}
(\boldsymbol{q} \times \boldsymbol{n}) \cdot \boldsymbol{D} & \equiv \mathbf{D}^{\top} \cdot(\boldsymbol{q} \times \boldsymbol{n}) \equiv \mathbf{e}_{k} \boldsymbol{d}_{k} \cdot(\boldsymbol{q} \times \boldsymbol{n}) \equiv \mathbf{e}_{k}\left(\boldsymbol{d}_{k} \times \boldsymbol{q}\right) \cdot \boldsymbol{n} \equiv\left(\mathbf{D}^{\top} \times \boldsymbol{q}\right) \cdot \boldsymbol{n} \\
& \equiv-\mathbf{e}_{k}\left(\boldsymbol{q} \times \boldsymbol{d}_{k}\right) \cdot \boldsymbol{n} \equiv-\boldsymbol{n} \cdot\left(\boldsymbol{q} \times \boldsymbol{d}_{k}\right) \mathbf{e}_{k} \equiv-\boldsymbol{n} \cdot(\boldsymbol{q} \times \boldsymbol{D})
\end{aligned}
$$

to isolate $\boldsymbol{n}_{0 t}(1, t)$ we write the rightmost integrals of (4.17) and (4.18) as

$$
\begin{aligned}
& \boldsymbol{E}(t) \cdot \boldsymbol{n}_{0 t}(1, t)+\boldsymbol{F}(t) \cdot \boldsymbol{m}_{0 t}(1, t), \\
& \boldsymbol{G}(t) \cdot \boldsymbol{n}_{0 t}(1, t)+\boldsymbol{H}(t) \cdot \boldsymbol{m}_{0 t}(1, t) .
\end{aligned}
$$


Let $\boldsymbol{a}, \boldsymbol{b}$ be arbitrary constant vectors in $\mathbb{E}^{3}$ and let

$$
\boldsymbol{a} \cdot \boldsymbol{D}_{0}(\xi, t)=: \mathbf{a}(\xi, t), \quad \boldsymbol{b} \cdot \boldsymbol{D}_{0}(\xi, t)=: \mathbf{b}(\xi, t), \quad \boldsymbol{a} \cdot\left(\boldsymbol{q} \times \boldsymbol{D}_{0}\right) \equiv-\left(\mathbf{D}_{0}^{\top} \times \boldsymbol{q}\right) \cdot \boldsymbol{a}=: \mathbf{h}(\xi, t) .
$$

Then the quadratic form for the matrix of coefficients of $\boldsymbol{n}_{0 t}(1, t)$ and $\boldsymbol{m}_{0 t}(1, t)$ in (4.20) is

$$
\begin{aligned}
& \boldsymbol{a} \cdot \boldsymbol{E} \cdot \boldsymbol{a}+\boldsymbol{a} \cdot \boldsymbol{F} \cdot \boldsymbol{b}+\boldsymbol{b} \cdot \boldsymbol{G} \cdot \boldsymbol{a}+\boldsymbol{b} \cdot \boldsymbol{H} \cdot \boldsymbol{b} \\
& =\int_{0}^{1}\left[\mathbf{a} \cdot \dot{\mathbf{v}}_{\mathbf{n}}^{\sharp} \cdot \mathbf{a}-\mathbf{a} \cdot \dot{\mathbf{v}}_{\mathbf{m}}^{\sharp} \cdot \mathbf{h}-\mathbf{h} \cdot \dot{\mathbf{u}}_{\mathbf{n}}^{\sharp} \cdot \mathbf{a}+\mathbf{h} \cdot \dot{\mathbf{u}}_{\mathbf{m}}^{\sharp} \cdot \mathbf{h}\right. \\
& \left.\quad \quad+\mathbf{a} \cdot \dot{\mathbf{v}}_{\mathbf{m}}^{\sharp} \cdot \mathbf{b}-\mathbf{h} \cdot \dot{\mathbf{u}}_{\mathbf{m}}^{\sharp} \cdot \mathbf{b}+\mathbf{b} \cdot \dot{\mathbf{u}}_{\mathbf{n}}^{\sharp} \cdot \mathbf{a}-\mathbf{b} \cdot \dot{\mathbf{u}}_{\mathbf{m}}^{\sharp} \cdot \mathbf{h}+\mathbf{b} \cdot \dot{\mathbf{u}}_{\mathbf{m}}^{\sharp} \cdot \mathbf{b}\right] d \xi \\
& =\int_{0}^{1}\left[\mathbf{a} \cdot \dot{\mathbf{v}}_{\mathbf{n}}^{\sharp} \cdot \mathbf{a}+\mathbf{a} \cdot \dot{\mathbf{v}}_{\mathbf{m}}^{\sharp} \cdot(\mathbf{b}-\mathbf{h})+(\mathbf{b}-\mathbf{h}) \cdot \dot{\mathbf{u}}_{\mathbf{n}}^{\sharp} \cdot \mathbf{a}+(\mathbf{b}-\mathbf{h}) \cdot \dot{\mathbf{u}}_{\mathbf{m}}^{\sharp} \cdot(\mathbf{b}-\mathbf{h})\right] d \xi .
\end{aligned}
$$

This expression is positive-definite by virtue of the monotonicity condition (2.36). Thus (4.17) and (4.18) can be uniquely solved for $\boldsymbol{m}_{0 t}(1, \cdot)$ and $\boldsymbol{n}_{0 t}(1, \cdot)$ in terms of the other variables. (This proof of the positive-definiteness of (4.22) corrects a gap in the corresponding proof of [3].)

The proof of existence and uniqueness of solutions of initial-value problems for this system of generalized ordinary differential equations is given in [3]. It uses the Contraction Mapping Principle to give the existence of solutions for a short time and uses a priori bounds to ensure existence for all time.

To simplify the terms of the initial-layer expansion, we shall use the reduced form of the compatibility equations (2.41) and (2.42):

$$
\begin{aligned}
\mathbf{v}_{0 t}+\mathbf{w}_{0} \times \mathbf{v}_{0} & =\mathbf{p}_{0 s}+\mathbf{u}_{0} \times \mathbf{p}_{0}, \\
\mathbf{u}_{0 t} & =\mathbf{w}_{0 s}+\mathbf{u}_{0} \times \mathbf{w}_{0} .
\end{aligned}
$$

5. Higher-order terms of the regular expansion. Substituting the regular expansion into the governing equations, differentiating these equations $j$ times with respect to $\varepsilon$, and setting $\varepsilon=0$, yields the $j$ th-order equations of the regular expansion. E.g., from (2.41), (2.42), and (2.39) we get

$$
\boldsymbol{v}_{j t}=\boldsymbol{p}_{j s}, \quad \boldsymbol{u}_{j t}=\boldsymbol{w}_{j s}+\sum_{l=0}^{j}\left(\begin{array}{l}
j \\
l
\end{array}\right) \boldsymbol{w}_{l} \times \boldsymbol{u}_{j-l}, \quad j \boldsymbol{p}_{(j-1) t}=\boldsymbol{n}_{j s},
$$

and from the constitutive equation $\boldsymbol{n}=\boldsymbol{D} \cdot \hat{\mathbf{n}}\left(\boldsymbol{\xi}, \boldsymbol{\xi}_{t}, s\right)$ we then get

$$
\boldsymbol{n}_{j}=\sum_{l=0}^{j}\left(\begin{array}{l}
j \\
l
\end{array}\right) \boldsymbol{D}_{l} \cdot \mathbf{n}_{j-l}
$$

with

$$
\begin{aligned}
\mathbf{n}_{1}= & \hat{\mathbf{n}}_{\boldsymbol{\xi}}\left(\boldsymbol{\xi}_{0}, \boldsymbol{\xi}_{0 t}, s\right) \cdot \boldsymbol{\xi}_{1}+\hat{\mathbf{n}}_{\dot{\boldsymbol{\xi}}}\left(\boldsymbol{\xi}_{0}, \boldsymbol{\xi}_{0 t}, s\right) \cdot \boldsymbol{\xi}_{1 t}, \\
\mathbf{n}_{2}= & \hat{\mathbf{n}}_{\boldsymbol{\xi}}\left(\boldsymbol{\xi}_{0}, \boldsymbol{\xi}_{0 t}, s\right) \cdot \boldsymbol{\xi}_{2}+\hat{\mathbf{n}}_{\dot{\boldsymbol{\xi}}}\left(\boldsymbol{\xi}_{0}, \boldsymbol{\xi}_{0 t}, s\right) \cdot \boldsymbol{\xi}_{2 t} \\
& +\hat{\mathbf{n}}_{\boldsymbol{\xi} \boldsymbol{\xi}}\left(\boldsymbol{\xi}_{0}, \boldsymbol{\xi}_{0 t}, s\right): \boldsymbol{\xi}_{1} \boldsymbol{\xi}_{1}+2 \hat{\mathbf{n}}_{\dot{\text { }}}\left(\boldsymbol{\xi}_{0}, \boldsymbol{\xi}_{0 t}, s\right): \boldsymbol{\xi}_{1} \boldsymbol{\xi}_{1 t}+\hat{\mathbf{n}}_{\dot{\boldsymbol{\xi}} \boldsymbol{\xi}}\left(\boldsymbol{\xi}_{0}, \boldsymbol{\xi}_{0 t}, s\right): \boldsymbol{\xi}_{1 t} \boldsymbol{\xi}_{1 t}, \\
\mathbf{n}_{j}= & \hat{\mathbf{n}}_{\boldsymbol{\xi}}\left(\boldsymbol{\xi}_{0}, \boldsymbol{\xi}_{0 t}, s\right) \cdot \boldsymbol{\xi}_{j}+\hat{\mathbf{n}}_{\dot{\boldsymbol{\xi}}}\left(\boldsymbol{\xi}_{0}, \boldsymbol{\xi}_{0 t}, s\right) \cdot \boldsymbol{\xi}_{j t}+\mathbf{n}_{j}^{-}
\end{aligned}
$$

where $\mathbf{n}_{j}^{-}$depends on $\boldsymbol{\xi}_{l}, \boldsymbol{\xi}_{l t}, l=0,1, \ldots, j-1$. 
In particular, we differentiate the system (2.39)-(2.44) and $(2.5)_{2}$ once with respect to $\varepsilon$, and set $\varepsilon=0$, to obtain the equations for the first perturbation of the regular expansion:

$$
\begin{aligned}
\rho A \boldsymbol{r}_{0 t t} & =\boldsymbol{n}_{1 s}, \\
\partial_{t}\left[\boldsymbol{D}_{0}(s, t) \cdot \rho \mathbf{I} \cdot \mathbf{w}_{0}(s, t)\right] & =\boldsymbol{m}_{1 s}+\boldsymbol{v}_{0} \times \boldsymbol{n}_{1}+\boldsymbol{v}_{1} \times \boldsymbol{n}_{0}, \\
\boldsymbol{D}_{1 t} & =\boldsymbol{w}_{1} \times \boldsymbol{D}_{0}+\boldsymbol{w}_{0} \times \boldsymbol{D}_{1}, \\
\boldsymbol{\xi}_{1 t} & =\dot{\boldsymbol{\xi}}_{\boldsymbol{\xi}}^{\sharp} \cdot \boldsymbol{\xi}_{1}+\dot{\boldsymbol{\xi}}_{\mathbf{n}}^{\sharp} \cdot \boldsymbol{n}_{1}+\dot{\boldsymbol{\xi}}_{\mathbf{m}}^{\sharp} \cdot \mathbf{m}_{1}, \\
\mu \boldsymbol{a}_{1}(1, t) & =-\boldsymbol{n}_{1}(1, t), \\
\boldsymbol{b}_{1}(1, t) & =-\boldsymbol{m}_{1}(1, t)+\boldsymbol{c}_{1}(t) \times \boldsymbol{n}_{0}(1, t)+\boldsymbol{c}_{0}(t) \times \boldsymbol{n}_{1}(1, t)
\end{aligned}
$$

where the arguments of the derivatives of $\dot{\boldsymbol{\xi}}^{\sharp}$ are the arguments of $\dot{\boldsymbol{\xi}}^{\sharp}$ shown in (4.6), and where

$$
\begin{aligned}
& \boldsymbol{n}_{1}:=\boldsymbol{D}_{1} \cdot \mathbf{n}_{0}+\boldsymbol{D}_{0} \cdot \mathbf{n}_{1}, \quad \text { etc. } \\
& \boldsymbol{a}_{1}(1, t):=\left\{\boldsymbol{r}_{1 t t}(1, t)+\boldsymbol{w}_{1 t}(1, t) \times \boldsymbol{c}_{0}(t)+\boldsymbol{w}_{0 t}(1, t) \times \boldsymbol{c}_{1}(t)\right. \\
& +\boldsymbol{w}_{1}(1, t) \times\left[\boldsymbol{w}_{0}(1, t) \times \boldsymbol{c}_{0}(t)\right]+\boldsymbol{w}_{0}(1, t) \times\left[\boldsymbol{w}_{1}(1, t) \times \boldsymbol{c}_{0}(t)\right] \\
& \left.+\boldsymbol{w}_{0}(1, t) \times\left[\boldsymbol{w}_{0}(1, t) \times \boldsymbol{c}_{1}(t)\right]\right\}, \\
& \boldsymbol{b}_{1}(1, t):=\left[\boldsymbol{D}_{1} \cdot \mathbf{J} \cdot \mathbf{w}_{0}(1, t)+\boldsymbol{D}_{0}(1, t) \cdot \mathbf{J} \cdot \mathbf{w}_{1}(1, t)\right]_{t}, \\
& \boldsymbol{c}_{1}(t):=\boldsymbol{D}_{1}(1, t) \cdot \mathbf{c} .
\end{aligned}
$$

We follow the scheme for the reduced problem: We integrate (5.4) with respect to $s$ and substitute the resulting formula for $\boldsymbol{n}_{1}$ into the integral of (5.5) with respect to $s$ to obtain

$$
\begin{aligned}
\boldsymbol{n}_{1}(s, t)=\tilde{\boldsymbol{n}}_{1}(s, t):=\boldsymbol{n}_{1}(1, t)-\int_{s}^{1}(\rho A)(\xi) \boldsymbol{r}_{0 t t}(\xi, t) d \xi \\
\boldsymbol{m}_{1}(s, t)=\tilde{\boldsymbol{m}}_{1}(s, t):=\boldsymbol{m}_{1}(1, t)+\int_{s}^{1} \boldsymbol{v}_{0}(\xi, t) \times\left[\boldsymbol{n}_{1}(1, t)-\int_{\xi}^{1}(\rho A)(\eta) \boldsymbol{r}_{0 t t}(\eta, t) d \eta\right] d \xi \\
\quad-\int_{s}^{1}\left\{\partial_{t}\left[\boldsymbol{D}_{0}(\xi, t) \cdot(\rho \mathbf{I})(\xi) \cdot \mathbf{w}_{0}(\xi, t)\right]+\boldsymbol{v}_{1}(\xi, t) \times \boldsymbol{n}_{0}(\xi, t)\right\} d \xi .
\end{aligned}
$$

We next substitute these representations into (5.7) to get

$$
\boldsymbol{\xi}_{1 t}=\dot{\boldsymbol{\xi}}_{\boldsymbol{\xi}}^{\sharp} \cdot \boldsymbol{\xi}_{1}+\dot{\boldsymbol{\xi}}_{\mathbf{n}}^{\sharp} \cdot \tilde{\mathbf{n}}_{1}+\dot{\boldsymbol{\xi}}_{\mathbf{m}}^{\sharp} \cdot \tilde{\mathbf{m}}_{1} .
$$

From $(2.6)_{1}$ and (2.12) we obtain

$$
\begin{aligned}
& \boldsymbol{r}_{1 s t}=\boldsymbol{D}_{1} \cdot \mathbf{v}_{0 t}+\boldsymbol{D}_{0} \cdot \mathbf{v}_{1 t}+\boldsymbol{w}_{1} \times \boldsymbol{r}_{0 s}+\boldsymbol{w}_{0} \times \boldsymbol{r}_{1 s}, \\
& \boldsymbol{w}_{1 s}=\boldsymbol{D}_{1} \cdot \mathbf{u}_{0 t}+\boldsymbol{D}_{0} \cdot \mathbf{u}_{1 t},
\end{aligned}
$$


whence

$$
\begin{aligned}
\boldsymbol{r}_{1 t}= & \int_{0}^{s}\left\{\boldsymbol{z}_{1} \times \boldsymbol{D}_{0} \cdot \mathbf{v}_{0 t}+\boldsymbol{D}_{0} \cdot \mathbf{v}_{1 t}\right. \\
& \left.\quad+\boldsymbol{w}_{1 s} \times\left[\boldsymbol{r}_{0}(s, t)-\boldsymbol{r}_{0}(\xi, t)\right]+\boldsymbol{D}_{0} \cdot \mathbf{u}_{0 t} \times\left[\boldsymbol{r}_{1}(s, t)-\boldsymbol{r}_{1}(\xi, t)\right]\right\} d \xi, \\
\boldsymbol{w}_{1}= & \int_{0}^{s}\left[\boldsymbol{D}_{1} \cdot \mathbf{u}_{0 t}+\boldsymbol{D}_{0} \cdot \mathbf{u}_{1 t}\right] d \xi
\end{aligned}
$$

where $\mathbf{v}_{1 t}$ and $\mathbf{u}_{1 t}$ are given by (5.15).

As in the preceding section, (5.6), (5.15), (5.17) form a system of generalized ordinary differential equations for $\boldsymbol{D}_{1}, \mathbf{v}_{1}, \mathbf{u}_{1}, \boldsymbol{r}_{1}$ provided that $\boldsymbol{n}_{1}(1, t)$ and $\boldsymbol{m}_{1}(1, t)$ are known. We now find equations for the $\boldsymbol{n}_{1}(1, t)$ and $\boldsymbol{m}_{1}(1, t)$. We first use (5.9) and (5.12) to express $\boldsymbol{w}_{1 t}(1, t)$ in terms of the other expressions in these equations. From (5.8), (5.9), (5.17) we obtain

$$
\begin{aligned}
- & \mu^{-1} \boldsymbol{n}_{1}(1, t)=\boldsymbol{r}_{1 t t}(1, t)+\cdots \\
= & \int_{0}^{1}\left\{\boldsymbol{D}_{0} \cdot \mathbf{v}_{1 t t}-\boldsymbol{q}(\xi, t) \times \boldsymbol{D}_{0} \cdot \mathbf{u}_{1 t t}+\cdots\right\} d \xi \\
= & \int_{0}^{1}\left\{\boldsymbol{D}_{0} \cdot\left[\dot{\mathbf{v}}_{\mathbf{n}}^{\sharp} \cdot \mathbf{D}^{\top}(\xi, t) \cdot \boldsymbol{n}_{1 t}(1, t)+\dot{\mathbf{v}}_{\mathbf{m}}^{\sharp} \cdot \mathbf{D}^{\top}(\xi, t) \cdot \boldsymbol{m}_{1 t}(1, t)\right]\right. \\
& \left.\quad+\boldsymbol{D}_{0} \cdot\left[\dot{\mathbf{u}}_{\mathbf{n}}^{\sharp} \cdot \mathbf{D}_{0}^{\top}(\xi, t) \cdot \boldsymbol{n}_{1 t}(1, t)+\dot{\mathbf{u}}_{\mathbf{m}}^{\sharp} \cdot \mathbf{D}^{\top}(\xi, t) \cdot \boldsymbol{m}_{1 t}(1, t)\right] \times \boldsymbol{q}(\xi, t)+\cdots\right\} d \xi \\
= & \int_{0}^{1}\left\{\boldsymbol{D}_{0}(\xi, t) \cdot \dot{\mathbf{v}}_{\mathbf{n}}^{\sharp}-\boldsymbol{q}(\xi, t) \times \boldsymbol{D}_{0}(\xi, t) \cdot \dot{\mathbf{u}}_{\mathbf{n}}^{\sharp}\right\} \cdot \mathbf{D}^{\top}(\xi, t) \cdot \boldsymbol{n}_{1 t}(1, t) d \xi \\
& +\int_{0}^{1}\left\{\boldsymbol{D}_{0}(\xi, t) \cdot \dot{\mathbf{v}}_{\mathbf{m}}^{\sharp}-\boldsymbol{q}(\xi, t) \times \boldsymbol{D}_{0}(\xi, t) \cdot \dot{\mathbf{u}}_{\mathbf{m}}^{\sharp}\right\} \cdot \mathbf{D}^{\top}(\xi, t) \cdot \boldsymbol{m}_{1 t}(1, t) d \xi+\cdots,
\end{aligned}
$$

where the ellipses contain terms not involving $\boldsymbol{n}_{1 t}(1, t)$ and $\boldsymbol{m}_{1 t}(1, t)$, and where the arguments of the derivatives of $\dot{\boldsymbol{\xi}}^{\sharp}$ are the arguments of $\dot{\boldsymbol{\xi}}^{\sharp}$ shown in (4.6). This equation and that coming from (5.14) (which is not shown) are virtually identical to the equations of (4.17). It immediately follows from the development of the preceding section that $\boldsymbol{n}_{1 t}(1, t)$ and $\boldsymbol{m}_{1 t}(1, t)$ are uniquely defined functions of the other variables, so that the first perturbation leads to a system of generalized ordinary differential equations. The existence and uniqueness of global solutions to initial-value problems follows from a simplified version of the proof of [3], briefly described at the end of the preceding section.

The equations governing higher-order perturbations of the regular expansion are just nonhomogeneous versions of the linear system treated in this section, with these nonhomogeneous terms depending on the variables of lower-order perturbations. The treatment of the global existence and uniqueness of their solutions thus follows immediately from that for the first perturbation.

To simplify the terms of the initial-layer expansion, we shall use the first perturbation of the regular expansion for the compatibility equations (2.41) and (2.42):

$$
\begin{aligned}
\mathbf{v}_{1 t}+\mathbf{w}_{1} \times \mathbf{v}_{0}+\mathbf{w}_{0} \times \mathbf{v}_{1} & =\mathbf{p}_{1 s}+\mathbf{u}_{1} \times \mathbf{p}_{0}+\mathbf{u}_{0} \times \mathbf{p}_{1}, \\
\mathbf{u}_{1 t} & =\mathbf{w}_{1 s}+\mathbf{u}_{1} \times \mathbf{w}_{0}+\mathbf{u}_{0} \times \mathbf{w}_{1} .
\end{aligned}
$$




\section{The leading term of initial-layer expansion. Set}

$$
\left(\rho \boldsymbol{I}_{0}\right)(s):=\boldsymbol{D}^{\circ}(s) \cdot \rho \mathbf{I}(s) \cdot \mathbf{D}^{\circ}(s)^{\top} .
$$

To get the leading terms of the initial-layer expansion (of order $j=0$ ), we simply take the limit as $\varepsilon \rightarrow 0$ in the stretched equations (2.51)-(2.57) and use (4.1), (4.2), (4.11):

$$
\begin{gathered}
\rho A \boldsymbol{p}_{0 \tau}^{\star} \equiv \rho A \boldsymbol{D}^{\circ} \cdot \mathbf{p}_{0 \tau}^{\star}=\boldsymbol{n}_{0 s}^{\star} \equiv \boldsymbol{n}_{0 s}^{\star}-\boldsymbol{n}_{0 s}(\cdot, 0), \\
\rho \boldsymbol{I}_{0} \cdot \boldsymbol{w}_{0 \tau}^{\star} \equiv \boldsymbol{D}^{\circ} \cdot \rho \mathbf{I} \cdot \mathbf{w}_{0 \tau}^{\star} \\
=\boldsymbol{m}_{0 s}^{\star}+\boldsymbol{v}^{\circ} \times \boldsymbol{n}_{0}^{\star} \equiv \boldsymbol{m}_{0 s}^{\star}-\boldsymbol{m}_{0 s}(\cdot, 0)+\boldsymbol{v}^{\circ} \times\left[\boldsymbol{n}_{0 s}^{\star}-\boldsymbol{n}_{0 s}(\cdot, 0)\right], \\
\boldsymbol{v}_{0 \tau}^{\star}=\boldsymbol{p}_{0 s}^{\star} \Longleftrightarrow \mathbf{v}_{0 \tau}^{\star}+\mathbf{w}_{0}^{\star} \times \mathbf{v}_{0}(\cdot, 0)=\mathbf{p}_{0 s}^{\star}+\mathbf{u}_{0}(\cdot, 0) \times \mathbf{p}_{0}^{\star}, \\
\boldsymbol{u}_{0 \tau}^{\star}=\boldsymbol{w}_{0 s}^{\star}+\boldsymbol{w}_{0}^{\star} \times \boldsymbol{u}^{\circ} \Longleftrightarrow \mathbf{u}_{0 \tau}^{\star}=\mathbf{w}_{0 s}^{\star}+\mathbf{u}_{0}(s \cdot, 0) \times \mathbf{w}_{0}^{\star}, \\
\boldsymbol{p}_{0 \tau}^{\star}(1, \tau)+\boldsymbol{w}_{0 \tau}^{\star}(1, \tau) \times \boldsymbol{c}_{0}^{\star}(\tau)=\boldsymbol{o} \quad \Longleftrightarrow \quad \mathbf{p}_{0 \tau}^{\star}(1, \tau)+\mathbf{w}_{0 \tau}^{\star}(1, \tau) \times \boldsymbol{D}^{\circ}(1) \cdot \mathbf{c}=\mathbf{o}, \\
{\left[\boldsymbol{J}_{0}^{\star}(\tau) \cdot \boldsymbol{w}_{0}^{\star}(1, \tau)\right]_{\tau} \equiv \boldsymbol{D}^{\circ}(1) \cdot \mathbf{J} \cdot \mathbf{w}_{0 \tau}^{\star}(1, \tau)=\boldsymbol{o} \Longrightarrow \mathbf{w}_{0 \tau}^{\star}(1, \tau)=\mathbf{o},} \\
\boldsymbol{p}_{0}^{\star}(0, \tau)=\boldsymbol{o}, \quad \boldsymbol{w}_{0}^{\star}(0, \tau)=\boldsymbol{o}, \\
\boldsymbol{p}_{0}(s, 0)+\boldsymbol{p}_{0}^{\star}(s, 0)=\boldsymbol{p}^{\circ}(s), \quad \boldsymbol{w}_{0}(s, 0)+\boldsymbol{w}_{0}^{\star}(s, 0)=\boldsymbol{w}^{\circ}(s)
\end{gathered}
$$

where (2.53) and (2.54) imply that

$$
\begin{aligned}
\boldsymbol{n}_{0}^{\star}(s, \tau) & =\boldsymbol{D}^{\circ}(s) \cdot \hat{\mathbf{n}}\left(\boldsymbol{\xi}^{\circ}(s), \lim _{\varepsilon \rightarrow 0}\left(\varepsilon^{-1} \boldsymbol{\xi}_{\tau}^{\star}(s, \tau)\right), s\right) \\
& \equiv \boldsymbol{D}^{\circ}(s) \cdot \hat{\mathbf{n}}\left(\boldsymbol{\xi}^{\circ}(s), \boldsymbol{\xi}_{0 t}(s, 0)+\boldsymbol{\xi}_{0 \tau}^{\star}(s, \tau), s\right),
\end{aligned}
$$

etc. We immediately conclude from (6.6), (6.7), and (6.9) that

$$
\mathbf{p}_{0}^{\star}(1, \tau)=\mathbf{p}_{0}^{\star}(1,0)=\mathbf{p}^{\circ}(1)-\mathbf{p}_{0}(1,0)=\mathbf{o}, \quad \mathbf{w}_{0}^{\star}(1, \tau)=\mathbf{w}_{0}^{\star}(1,0)=\mathbf{w}^{\circ}(1)-\mathbf{w}_{0}(1,0)=\mathbf{o}
$$

by (4.9). Our constitutive assumptions ensure that (6.2), 6.3), 6.10) form a quasilinear semimonotone parabolic system for $\mathbf{p}_{0}^{\star}$ and $\mathbf{w}_{0}^{\star}$. We discuss its solvability in Section 8 ,

7. Higher-order terms of the initial-layer expansion. To get the $j$ th-order terms of the initial-layer expansion we substitute (3.7) and (3.9) into the stretched equations (2.51) - (2.57), noting the difference between the two formulas in (3.4), differentiate these equations $j$ times with respect to $\varepsilon$, set $\varepsilon=0$, use generalizations of the formulas (3.8) and (3.10), and simplify the resulting equations by using the corresponding equations for the regular expansion. E.g., the substitution of (3.7) and (3.9) into (2.53) (the stretched equation, simplest because it is linear) yields

$$
\begin{aligned}
\varepsilon^{-1} \partial_{\tau} \boldsymbol{v}_{\mathrm{A}}^{H}(s, \varepsilon \tau ; \varepsilon) & \equiv \sum_{j=0}^{H} \boldsymbol{v}_{j t}(s, \varepsilon \tau) \frac{\varepsilon^{j}}{j !}+\sum_{j=0}^{H-1} \boldsymbol{v}_{j \tau}^{\star}(s, \tau) \frac{\varepsilon^{j}}{(j+1) !} \\
& =\sum_{j=0}^{H} \boldsymbol{p}_{j s}(s, \varepsilon \tau) \frac{\varepsilon^{j}}{j !}+\sum_{j=0}^{H} \boldsymbol{p}_{j s}^{\star}(s, \tau) \frac{\varepsilon^{j}}{j !} \equiv \partial_{s} \boldsymbol{p}_{\mathrm{A}}^{H}(s, \varepsilon \tau ; \varepsilon) .
\end{aligned}
$$


Differentiating (7.1) $q$ times with respect to $\varepsilon$ yields

$$
\begin{aligned}
& \sum_{j=0}^{H} \sum_{h=0}^{q} \tau^{h} \partial_{t}^{h+1} \boldsymbol{v}_{j}(s, \varepsilon \tau) \frac{\varepsilon^{j-q+h}}{(j-q+h) !}+\sum_{j=0}^{H-1} \boldsymbol{v}_{j \tau}^{\star}(s, \tau) \frac{j ! \varepsilon^{j-q}}{(j-q) !(j+1) !} \\
& =\sum_{j=0}^{H} \sum_{h=0}^{q} \tau^{h} \partial_{t}^{h} \boldsymbol{p}_{j s}(s, \varepsilon \tau) \frac{\varepsilon^{j-q+h}}{(j-q+h) !}+\sum_{j=0}^{H} \boldsymbol{p}_{j s}^{\star}(s, \tau) \frac{\varepsilon^{j-q}}{(j-q) !} .
\end{aligned}
$$

This equation evaluated at $\varepsilon=0$ is

$$
\sum_{h=0}^{q} \tau^{h} \partial_{t}^{h} \boldsymbol{v}_{(q+h) t}(s, 0)+\frac{\boldsymbol{v}_{q \tau}^{\star}(s, \tau)}{j+1}=\sum_{h=0}^{q} \tau^{h} \partial_{t}^{h} \boldsymbol{p}_{(q+h) s}(s, 0)+\boldsymbol{p}_{q s}^{\star}(s, \tau),
$$

which $(5.1)_{1}$ reduces to

$$
\boldsymbol{v}_{q \tau}^{\star}(s, \tau)=(j+1) \boldsymbol{p}_{q s}^{\star}(s, \tau)
$$

In particular, the first-order equations of the initial-layer expansion are

$$
\begin{gathered}
\rho A\left[\boldsymbol{p}_{0 t}(s, 0)+\boldsymbol{p}_{1 \tau}^{\star}\right]=\boldsymbol{n}_{1 s}^{\star} \\
\left\{\left[\tau \boldsymbol{D}_{0 t}(s, 0)+\boldsymbol{D}_{1}(s, 0)+\boldsymbol{D}_{0}^{\star}(s, \tau)\right] \cdot \rho \mathbf{I} \cdot\left[\mathbf{w}_{0}(s, 0)+\mathbf{w}_{0}^{\star}(s, \tau)\right]\right\}_{\tau} \\
\quad+\left\{\boldsymbol{D}^{\circ}(s) \cdot \rho \mathbf{I} \cdot\left[\tau \mathbf{w}_{0 t}(s, 0)+\mathbf{w}_{1}(s, 0)+\mathbf{w}_{1}^{\star}(s, \tau)\right]\right\}_{\tau} \\
=\boldsymbol{m}_{1 s}^{\star}+\left[\tau \boldsymbol{v}_{0 t}(s, 0)+\boldsymbol{v}_{1}(s, 0)+\boldsymbol{v}_{0}^{\star}(s, \tau)\right] \times \boldsymbol{n}_{0}^{\star}+\boldsymbol{v}^{\circ}(s) \times \boldsymbol{n}_{1}^{\star}, \\
\mathbf{v}_{1 t}^{\star}+2 \tau \mathbf{v}_{0 t t}(s, 0)+2 \mathbf{v}_{1 t}(s, 0) \\
=-2\left[\tau \mathbf{w}_{0 t}(s, 0)+\mathbf{w}_{1}(s, 0)+\mathbf{w}_{1}^{\star}\right] \times \mathbf{v}_{0}(s, 0) \\
\left.-2\left[\mathbf{w}_{0}(s, 0)\right)+\mathbf{w}_{0}^{\star}\right] \times\left[\tau \mathbf{v}_{0 t}(s, 0)+\mathbf{v}_{1}(s, 0)+\mathbf{v}_{0}^{\star}\right] \\
+2\left[\tau \mathbf{p}_{0 s t}(s, 0)+\mathbf{p}_{1 s}(s, 0)+\mathbf{p}_{1 s}^{\star}\right]+2\left[\tau \mathbf{u}_{0 t}(s, 0)+\mathbf{u}_{1}(s, 0)+\mathbf{u}_{0}^{\star}\right] \times\left[\mathbf{p}_{0}(s, 0)+\mathbf{p}_{0}^{\star}\right] \\
+2 \mathbf{u}^{\circ}(s) \times\left[\tau \mathbf{p}_{0 t}(s, 0)+\mathbf{p}_{1}(s, 0)+\mathbf{p}_{1}^{\star}\right] \\
=: 2 \dot{\mathbf{v}}^{\mathrm{b}}, \\
\mathbf{u}_{1 \tau}^{\star}+2 \mathbf{u}_{1 t}(s, 0)+2 \tau \mathbf{u}_{0 t t}(s, 0) \\
=2\left[\tau \mathbf{w}_{0 s t}(s, 0)+\mathbf{w}_{1 s}(s, 0)+\mathbf{w}_{1 s}^{\star}\right]+2 \mathbf{u}^{\circ}(s) \times\left[\tau \mathbf{w}_{0 t}(s, 0)+\mathbf{w}_{1}(s, 0)+\mathbf{w}_{1}^{\star}\right] \\
+2\left[\tau \mathbf{u}_{0 t}(s, 0)+\mathbf{u}_{1}(s, 0)+\mathbf{u}_{0}^{\star}\right] \times\left[\mathbf{w}_{0}(s, 0)+\mathbf{w}_{0}^{\star}\right] \\
=: 2 \dot{\mathbf{u}}^{b}
\end{gathered}
$$

where

$$
\begin{aligned}
\boldsymbol{n}_{1}^{\star}(s, \tau)=\left[\tau \boldsymbol{D}_{0 t}(s, 0)+\boldsymbol{D}_{1}(s, 0)+\boldsymbol{D}_{0}^{\star}(s, \tau)\right] \cdot \mathbf{D}^{\circ}(s)^{\top} \cdot \boldsymbol{n}_{0}^{\star}(s, \tau) \\
+\boldsymbol{D}^{\circ}(s) \cdot\left\{\hat{\mathbf{n}}_{\mathbf{v}} \cdot\left[\tau \mathbf{v}_{0 t}(s, 0)+\mathbf{v}_{1}(s, 0)+\mathbf{v}_{0}^{\star}(s, \tau)\right]\right. \\
+\hat{\mathbf{n}}_{\mathbf{u}} \cdot\left[\tau \mathbf{u}_{0 t}(s, 0)+\mathbf{u}_{1}(s, 0)+\mathbf{u}_{0}^{\star}(s, \tau)\right] \\
\left.+\hat{\mathbf{n}}_{\dot{\mathbf{v}}} \cdot \dot{\mathbf{v}}^{b}+\hat{\mathbf{n}}_{\dot{\mathbf{u}}} \cdot \dot{\mathbf{u}}^{b}\right\}, \quad \text { etc. },
\end{aligned}
$$

in which the arguments of the derivatives of $\hat{\mathbf{n}}$ and $\hat{\mathbf{m}}$ are those shown in (6.10).

Now (2.55) is

$\mu\left\{\boldsymbol{p}_{\tau}^{\star}(1, \tau)+\boldsymbol{w}_{\tau}^{\star}(1, \tau) \times \boldsymbol{D}^{\star}(1, \tau) \cdot \mathbf{c}+\varepsilon \boldsymbol{w}^{\star}(1, \tau) \times\left[\boldsymbol{w}^{\star}(1, \tau) \times \boldsymbol{D}^{\star}(1, \tau) \cdot \mathbf{c}\right]\right\}=-\varepsilon \boldsymbol{n}^{\star}(1, t)$. 
The $\varepsilon$-derivative of this equation at $\varepsilon=0$ is

$$
\begin{aligned}
\mu & \left\{\boldsymbol{p}_{0 t}(1,0)+\boldsymbol{p}_{1 \tau}^{\star}(1, \tau)+\left[\boldsymbol{w}_{0 t}(1,0)+\boldsymbol{w}_{1 \tau}^{\star}(1, \tau)\right] \times \boldsymbol{D}^{\circ}(1) \cdot \mathbf{c}\right. \\
& +\boldsymbol{w}_{0 \tau}^{\star}(1, \tau) \times\left[\tau \boldsymbol{D}_{0 t}(1,0)+\boldsymbol{D}_{1}(1,0)+\boldsymbol{D}_{0}^{\star}(1, \tau)\right] \cdot \mathbf{c} \\
& \left.+\left(\boldsymbol{w}_{0}(1,0)+\boldsymbol{w}_{0}^{\star}(1, \tau)\right) \times\left[\left(\boldsymbol{w}_{0}(1,0)+\boldsymbol{w}_{0}^{\star}(1, \tau)\right) \times \boldsymbol{D}^{\circ}(1) \cdot \mathbf{c}\right]\right\} \\
= & -\boldsymbol{D}^{\circ}(1) \cdot \hat{\mathbf{n}}\left(\boldsymbol{\xi}_{0}(1,0), \boldsymbol{\xi}_{0 t}(1,0)+\boldsymbol{\xi}_{0 \tau}^{\star}(1, \tau), 1\right) .
\end{aligned}
$$

The use of (6.11) reduces this equation to

$$
\begin{aligned}
& \mu\left\{\boldsymbol{p}_{0 t}(1,0)+\boldsymbol{p}_{1 \tau}^{\star}(1, \tau)+\left[\boldsymbol{w}_{0 t}(1,0)+\boldsymbol{w}_{1 \tau}^{\star}(1, \tau)\right] \times \boldsymbol{D}^{\circ}(1) \cdot \mathbf{c}\right. \\
& \left.\quad+\boldsymbol{w}_{0}(1,0) \times\left[\boldsymbol{w}_{0}(1,0) \times \boldsymbol{D}^{\circ}(1) \cdot \mathbf{c}\right]\right\} \\
& =-\boldsymbol{D}^{\circ}(1) \cdot \hat{\mathbf{n}}\left(\boldsymbol{\xi}_{0}(1,0), \boldsymbol{\xi}_{0 t}(1,0)+\boldsymbol{\xi}_{0 \tau}^{\star}(1, \tau), 1\right) .
\end{aligned}
$$

We combine (7.12) with (4.3) and use (6.4) and (6.5) to obtain

$$
\begin{aligned}
\mu \boldsymbol{p}_{1 \tau}^{\star}(1, \tau)= & \boldsymbol{D}^{\circ}(1) \cdot \hat{\mathbf{n}}\left(\boldsymbol{\xi}_{0}(1,0), \boldsymbol{\xi}_{0 t}(1,0), 1\right) \\
& -\boldsymbol{D}^{\circ}(1) \cdot \hat{\mathbf{n}}\left(\boldsymbol{\xi}_{0}(1,0), \boldsymbol{\xi}_{0 t}(1,0)+\boldsymbol{\xi}_{0 \tau}^{\star}(1, \tau), 1\right) \\
\equiv & \boldsymbol{D}^{\circ}(1) \cdot \hat{\mathbf{n}}\left(\boldsymbol{\xi}_{0}(1,0), \boldsymbol{\xi}_{0 t}(1,0), 1\right) \\
& -\boldsymbol{D}^{\circ}(1) \cdot \hat{\mathbf{n}}\left(\boldsymbol{\xi}_{0}(1,0), \mathbf{v}_{0 t}(1,0)+\mathbf{p}_{0 s}^{\star}(1, \tau), \mathbf{u}_{0 t}(1,0)+\mathbf{w}_{0 s}^{\star}(1, \tau), 1\right) .
\end{aligned}
$$

All the equations of this section can be simplified somewhat: E.g., the substitution of (4.23) and (5.19) into (7.8) reduces it to

$$
\mathbf{u}_{1 \tau}^{\star}=2 \mathbf{w}_{1 s}^{\star}+2 \mathbf{u}_{0}^{\star} \times \mathbf{w}_{0}(\cdot, 0)+2\left[\tau \mathbf{u}_{0 t}(\cdot, 0)+\mathbf{u}_{1}(\cdot, 0)+\mathbf{u}_{0}^{\star}\right] \times \mathbf{w}_{0}^{\star}+2 \mathbf{u}_{0}(\cdot, 0) \times \mathbf{w}_{1}^{\star} .
$$

System (7.5) - (7.9) is typical of all the systems for the higher-order terms of the initiallayer expansion, which are nonhomogeneous versions of the same linear parabolic system. The $k$ th-order terms of the initial-layer expansion depend on the terms of the initial-layer expansion of orders 0 to $k-1$ and on terms of the regular expansion of orders 0 to $k$.

8. Exponential decay of the initial-layer expansion. In this section we use a version of the Faedo-Galerkin method [14, 23] (akin to that used by [4] for existence theory) to get exponential decay of the terms of the initial-layer expansion. The tricky estimates that we obtain support a standard proof by this method of the existence and regularity of the solutions of the initial-boundary-value problems of the initial-layer expansion (which are strong semi-monotone parabolic systems). We comment on this matter below.

Expansion functions. For functions $\boldsymbol{f}, \boldsymbol{g} \in\left[L^{2}(0,1)\right]^{3}, \zeta \in L^{2}(0,1)$ set

$$
\langle\boldsymbol{f}, \boldsymbol{g}\rangle:=\int_{0}^{1} \boldsymbol{f}(s) \cdot \boldsymbol{g}(s) d s, \quad\|\boldsymbol{f}\|:=\sqrt{\langle\boldsymbol{f}, \boldsymbol{f}\rangle}, \quad\langle\boldsymbol{f}, \zeta\rangle:=\int_{0}^{1} \boldsymbol{f}(s) \zeta(s) d s,
$$

etc. Our Faedo-Galerkin method uses the simplest expansion functions:

$$
x_{k}(s)=\sqrt{2} \cos k \pi s, \quad y_{k}(s):=\sqrt{2} \sin k \pi s, \quad k=1,2, \ldots,
$$


which satisfy

$$
\begin{gathered}
\left\langle x_{k}, x_{l}\right\rangle=\delta_{k l}=\left\langle y_{k}, y_{l}\right\rangle \\
x_{k s}=-k \pi y_{k}, \quad y_{k s}=k \pi x_{k}, \quad \int_{0}^{s} x_{k}(\xi) d \xi=\frac{y_{k}}{k \pi}, \quad \int_{0}^{s} y_{k}(\xi) d \xi=\frac{\sqrt{2}}{k \pi}-\frac{x_{k}}{k \pi}
\end{gathered}
$$

where $\delta_{k l}$ is the Kronecker delta. Define the orthogonal projectors $\boldsymbol{X}^{K}$ and $\boldsymbol{Y}^{K}$ of squareintegrable vector-valued functions $\boldsymbol{f}$ respectively onto linear combinations of $\left\{x_{1}, \ldots, x_{K}\right\}$ and linear combinations of $\left\{y_{1}, \ldots, y_{K}\right\}$ by

$$
\boldsymbol{X}^{K} \cdot \boldsymbol{f}:=\sum_{k=0}^{K}\left\langle\boldsymbol{f}, x_{k}\right\rangle x_{k}, \quad \text { etc. }
$$

so that

$$
\left\langle\boldsymbol{f}, \boldsymbol{X}^{K} \cdot \boldsymbol{g}\right\rangle \equiv\left\langle\boldsymbol{X}^{K} \cdot \boldsymbol{f}, \boldsymbol{g}\right\rangle \equiv\left\langle\boldsymbol{X}^{K} \cdot \boldsymbol{f}, \boldsymbol{X}^{K} \cdot \boldsymbol{g}\right\rangle, \quad\left\|\boldsymbol{X}^{K} \boldsymbol{f}\right\| \leq\|\boldsymbol{f}\|, \quad \text { etc. }
$$

Assumption (2.19) implies that there are positive constants $c, C$ such that

$$
c\|\boldsymbol{f}\|^{2} \leq\langle\rho A \boldsymbol{f}, \boldsymbol{f}\rangle \leq C\|\boldsymbol{f}\|^{2}, \quad c\|\boldsymbol{f}\|^{2} \leq\left\langle\rho \boldsymbol{I}_{0} \boldsymbol{f}, \boldsymbol{f}\right\rangle \leq C\|\boldsymbol{f}\|^{2} .
$$

Convention on constants. In the rest of the paper we adopt the convention that $C$ and $c$ denote typical positive constants (with $C$ large and $c$ small) that are supplied as data or that can be estimated in terms of data. Their meanings often change with each appearance (even in the same equation or inequality). A statement of the form $g \leq C$ means that there exists a $C$ such that this inequality holds, etc.

Inequalities. We use without comment the Cauchy-Bunyakovskiü-Schwarz inequality, which states that $|\langle\boldsymbol{f}, \boldsymbol{g}\rangle| \leq\|\boldsymbol{f}\|\|\boldsymbol{g}\|$, and the elementary inequality $2 \alpha \beta \leq(\alpha / \gamma)^{2}+(\beta \gamma)^{2}$ for all $\alpha, \beta, \gamma \in \mathbb{R}$ with $\gamma \neq 0$. In accord with the convention on constants, we often write this inequality as $\alpha \beta \leq C \alpha^{2}+c \beta^{2}$.

The weak formulation. A standard weak form of the initial-boundary-value problem (6.2) - 6.11) for $\boldsymbol{p}_{0}^{\star}, \boldsymbol{w}_{0}^{\star}$ (with the components of $\boldsymbol{p}_{0}^{\star}(\cdot, \tau), \boldsymbol{w}_{0}^{\star}(\cdot, \tau)$ in the Sobolev space $H_{0}^{1}(0,1) \equiv\left\{y: y_{s} \in L^{2}(0,1), y(0)=0=y(1)\right\}$ so that the boundary conditions (6.11) are satisfied) is

$$
\begin{gathered}
\left\langle\rho A \boldsymbol{p}_{0 \tau}^{\star}, y\right\rangle=-\left\langle\boldsymbol{n}_{0}^{\star}, y_{s}\right\rangle \equiv-\left\langle\boldsymbol{n}_{0}^{\star}-\boldsymbol{n}_{0}, y_{s}\right\rangle, \\
\left\langle\rho \boldsymbol{I}_{0} \cdot \boldsymbol{w}_{0 \tau}^{\star}, y\right\rangle=-\left\langle\boldsymbol{m}_{0}^{\star}, y_{s}\right\rangle+\left\langle\boldsymbol{v}^{\circ} \times \boldsymbol{n}_{0}^{\star}, y\right\rangle \equiv-\left\langle\boldsymbol{m}_{0}^{\star}-\boldsymbol{m}_{0}, y_{s}\right\rangle+\left\langle\boldsymbol{v}^{\circ} \times\left(\boldsymbol{n}_{0}^{\star}-\boldsymbol{n}_{0}\right), y\right\rangle, \\
\boldsymbol{p}_{0}(s, 0)+\boldsymbol{p}_{0}^{\star}(s, 0)=\boldsymbol{p}^{\circ}(s), \quad \boldsymbol{w}_{0}(s, 0)+\boldsymbol{w}_{0}^{\star}(s, 0)=\boldsymbol{w}^{\circ}(s)
\end{gathered}
$$

for all $y \in H_{0}^{1}(0,1)$, where

$$
\begin{gathered}
\boldsymbol{n}_{0}^{\star}(s, \tau)=\boldsymbol{D}^{\circ}(s) \cdot \hat{\mathbf{n}}\left(\boldsymbol{\xi}^{\circ}(s), \boldsymbol{\xi}_{0 t}(s, 0)+\boldsymbol{\xi}_{0 \tau}^{\star}(s, \tau), s\right), \quad \text { etc. }, \\
\boldsymbol{v}_{0 \tau}^{\star}=\boldsymbol{p}_{0 s}^{\star} \Longleftrightarrow \mathbf{v}_{0 \tau}^{\star}+\mathbf{w}_{0}^{\star} \times \mathbf{v}_{0}(s, 0)=\mathbf{p}_{0 s}^{\star}+\mathbf{u}_{0}(s, 0) \times \mathbf{p}_{0}^{\star}, \\
\boldsymbol{u}_{0 \tau}^{\star}=\boldsymbol{w}_{0 s}^{\star}+\boldsymbol{w}_{0}^{\star} \times \boldsymbol{u}^{\circ}(s) \Longleftrightarrow \mathbf{u}_{0 \tau}^{\star}=\mathbf{w}_{0 s}^{\star}+\mathbf{u}_{0}(s, 0) \times \mathbf{w}_{0}^{\star} .
\end{gathered}
$$


Identities. We first obtain some identities for the leading term of the initial-layer expansion. In view of Section 3 , the limits as $\varepsilon \rightarrow 0$ of the equations

$$
\begin{aligned}
\varepsilon^{-1} \boldsymbol{v}_{\tau}^{\star} & \equiv \varepsilon^{-1}\left(\boldsymbol{D}^{\star} \cdot \mathbf{v}^{\star}\right)_{\tau}=\varepsilon^{-1} \boldsymbol{D}_{\tau}^{\star} \cdot \mathbf{v}^{\star}+\varepsilon^{-1} \boldsymbol{D}^{\star} \cdot \mathbf{v}_{\tau}^{\star}, \\
\varepsilon^{-1} \boldsymbol{u}_{\tau}^{\star} & \equiv \varepsilon^{-1}\left(\boldsymbol{D}^{\star} \cdot \mathbf{u}^{\star}\right)_{\tau}=\boldsymbol{w}^{\star} \times \boldsymbol{D}^{\star} \cdot \mathbf{u}^{\star}+\varepsilon^{-1} \boldsymbol{D}^{\star} \cdot \mathbf{u}_{\tau}^{\star}, \\
\varepsilon^{-1} \boldsymbol{D}_{\tau} & =\boldsymbol{w} \times \boldsymbol{D}, \\
\boldsymbol{w}_{s}^{\star} & =\boldsymbol{D}^{\star} \cdot \varepsilon^{-1} \mathbf{u}_{\tau}^{\star}
\end{aligned}
$$

(which come from (2.53), (2.54), (2.5), (2.12)) are

$$
\begin{aligned}
\boldsymbol{v}_{0 t}(s, 0)+\boldsymbol{v}_{0 \tau}^{\star}(s, \tau)= & {\left[\boldsymbol{w}_{0}(s, 0)+\boldsymbol{w}_{0}^{\star}(s, \tau)\right] \times \boldsymbol{D}^{\circ}(s) \cdot \mathbf{v}_{0}(s, 0) } \\
& +\boldsymbol{D}^{\circ}(s) \cdot\left[\mathbf{v}_{0 t}(s, 0)+\mathbf{v}_{0 \tau}^{\star}(s, \tau)\right] \\
\Longrightarrow \quad \boldsymbol{v}_{0 \tau}^{\star}(s, \tau)= & \boldsymbol{w}_{0}^{\star}(s, \tau) \times \boldsymbol{D}^{\circ}(s) \cdot \mathbf{v}_{0}(s, 0)+\boldsymbol{D}^{\circ}(s) \cdot \mathbf{v}_{0 \tau}^{\star}(s, \tau), \\
\boldsymbol{u}_{0 t}(s, 0)+\boldsymbol{u}_{0 \tau}^{\star}(s, \tau)= & {\left[\boldsymbol{w}_{0}(s, 0)+\boldsymbol{w}_{0}^{\star}(s, \tau)\right] \times \boldsymbol{D}^{\circ}(s) \cdot \mathbf{u}_{0}(s, 0) } \\
& +\boldsymbol{D}^{\circ}(s) \cdot\left[\mathbf{u}_{0 t}(s, 0)+\mathbf{u}_{0 \tau}^{\star}(s, \tau)\right], \\
\Longrightarrow \quad \boldsymbol{u}_{0 \tau}^{\star}(s, \tau)= & \boldsymbol{w}_{0}^{\star}(s, \tau) \times \boldsymbol{D}^{\circ}(s) \cdot \mathbf{u}_{0}(s, 0)+\boldsymbol{D}^{\circ}(s) \cdot \mathbf{u}_{0 \tau}^{\star}(s, \tau), \\
\boldsymbol{D}_{0 t}(s, 0)+\boldsymbol{D}_{0 \tau}^{\star}(s, \tau)= & {\left[\boldsymbol{w}_{0}(s, 0)+\boldsymbol{w}_{0}^{\star}(s, \tau)\right] \times \boldsymbol{D}^{\circ}(s) } \\
\Longrightarrow \quad \boldsymbol{D}_{0 \tau}^{\star}(s, \tau)= & \boldsymbol{w}_{0}^{\star}(s, \tau) \times \boldsymbol{D}^{\circ}(s), \\
\mathbf{w}_{0 s}(s, 0)+\mathbf{w}_{0 s}^{\star}(s, \tau)= & \boldsymbol{D}^{\circ}(s) \cdot\left[\mathbf{u}_{0 t}(s, 0)+\mathbf{u}_{0 \tau}^{\star}(s, \tau)\right] \\
\Longrightarrow \quad \mathbf{w}_{0 s}^{\star}(s, \tau)= & \boldsymbol{D}^{\circ}(s) \cdot \mathbf{u}_{0 \tau}^{\star}(s, \tau) .
\end{aligned}
$$

Galerkin approximations. We seek Galerkin approximations of the solutions of (8.7) in the form

$$
\begin{aligned}
& \boldsymbol{p}_{0}^{\star}(s, \tau) \sim \boldsymbol{p}^{K}(s, \tau):=\sum_{k=1}^{K} \boldsymbol{p}_{k}(\tau) y_{k}(s), \\
& \boldsymbol{w}_{0}^{\star}(s, \tau) \sim \boldsymbol{w}^{K}(s, \tau):=\sum_{k=1}^{K} \boldsymbol{w}_{k}(\tau) y_{k}(s), \\
& \boldsymbol{v}_{0}^{\star}(s, \tau) \sim \boldsymbol{v}^{K}(s, \tau):=\int_{0}^{\tau} \boldsymbol{p}_{s}^{K}(s, \sigma) d \sigma, \\
& \boldsymbol{u}_{0}^{\star}(s, \tau) \sim \boldsymbol{u}^{K}(s, \tau):=\int_{0}^{\tau}\left[\boldsymbol{w}_{s}^{K}(s, \sigma)+\boldsymbol{w}^{K}(s, \sigma) \times \boldsymbol{u}^{\circ}(s)\right] d \sigma .
\end{aligned}
$$

(The coefficients $\boldsymbol{p}_{k}$ and $\boldsymbol{w}_{k}$ depend on $K$, but we do not clutter the notation further to indicate this fact.) In accord with (8.12) 2,4 we define $\mathbf{v}^{K}$ and $\mathbf{u}^{K}$ as solutions of

$$
\boldsymbol{p}_{s}^{K} \equiv \boldsymbol{v}_{\tau}^{K}=\boldsymbol{D}^{\circ} \cdot \mathbf{v}_{\tau}^{K}+\boldsymbol{w}^{K} \times \boldsymbol{v}^{\circ}, \quad \boldsymbol{u}_{\tau}^{K}=\boldsymbol{D}^{\circ} \cdot \mathbf{u}_{\tau}^{K}+\boldsymbol{w}^{K} \times \boldsymbol{u}^{\circ}
$$

subject to initial conditions coming from (8.13). Equation (8.12) 8 leads to the identity:

$$
\boldsymbol{w}_{s}^{K}(s, \tau):=\boldsymbol{D}^{\circ}(s) \cdot \mathbf{u}_{\tau}^{K}(s, \tau)
$$

(which also follows from $\left({ }^{8.13}\right)_{4}$ and $\left.(8.14)_{2}\right)$. Let

$$
\boldsymbol{n}^{K} \equiv \boldsymbol{D}^{\circ} \cdot \mathbf{n}^{K} \quad \text { where } \quad \mathbf{n}^{K}:=\hat{\mathbf{n}}\left(\boldsymbol{\xi}_{0}(\cdot, 0), \boldsymbol{\xi}_{0 t}(\cdot, 0)+\boldsymbol{\xi}_{\tau}^{K}, \cdot\right)-\hat{\mathbf{n}}\left(\boldsymbol{\xi}_{0}(\cdot, 0), \boldsymbol{\xi}_{0 t}(\cdot, 0), \cdot\right),
$$


etc. The substitution of (8.13) -8.15) into (8.7) with $\boldsymbol{n}_{0}^{\star}-\boldsymbol{n}_{0}$ and $\boldsymbol{m}_{0}^{\star}-\boldsymbol{m}_{0}$ replaced by $\boldsymbol{n}^{K}$ and $\boldsymbol{m}^{K}$ and with $y$ replaced by $y_{l}$ and the use of (8.5) yield a system of ordinary differential equations

$$
\begin{gathered}
\left\langle\rho A \boldsymbol{p}_{\tau}^{K}, y_{k}\right\rangle=-k \pi\left\langle\boldsymbol{n}^{K}, x_{k}\right\rangle \equiv-\left\langle\boldsymbol{n}^{K}, y_{k s}\right\rangle, \quad k=1,2, \ldots, K, \\
\left\langle\rho \boldsymbol{I}_{0} \cdot \boldsymbol{w}_{\tau}^{K}, y_{k}\right\rangle=-k \pi\left\langle\boldsymbol{m}^{K}, x_{k}\right\rangle+\left\langle\boldsymbol{v}^{\circ} \times \boldsymbol{n}^{K}, y_{k}\right\rangle, \quad k=1,2, \ldots, K,
\end{gathered}
$$

or, equivalently,

$$
\begin{aligned}
\left\langle\boldsymbol{Y}^{K} \cdot \rho A \boldsymbol{p}_{\tau}^{K}, y_{k}\right\rangle & =-k \pi\left\langle\boldsymbol{X}^{K} \cdot \boldsymbol{n}^{K}, x_{k}\right\rangle, \\
\left\langle\boldsymbol{Y}^{K} \cdot \rho \boldsymbol{I}_{0} \cdot \boldsymbol{w}_{\tau}^{K}, y_{k}\right\rangle & =-k \pi\left\langle\boldsymbol{X}^{K} \cdot \boldsymbol{m}^{K}, x_{k}\right\rangle+\left\langle\boldsymbol{Y}^{K} \cdot\left[\boldsymbol{v}^{\circ} \times \boldsymbol{n}^{K}\right], y_{k}\right\rangle .
\end{aligned}
$$

(Note that these equations hold for all $k$.)

$L^{2}$ bounds for $\boldsymbol{p}^{K}$ and $\boldsymbol{w}^{K}$. Taking the dot products of the equations of (8.17) respectively with $\boldsymbol{p}_{k}$ and $\boldsymbol{w}_{k}$, summing the resulting products over $k$, and using (8.14), (8.15), and (2.33), we obtain an energy equation and inequality:

$$
\begin{aligned}
& \frac{1}{2} \frac{d}{d \tau}\left[\left\langle\rho A \boldsymbol{p}^{K}, \boldsymbol{p}^{K}\right\rangle+\left\langle\rho \boldsymbol{I}_{0} \cdot \boldsymbol{w}^{K}, \boldsymbol{w}^{K}\right\rangle\right] \\
& =-\left\langle\boldsymbol{n}^{K}, \boldsymbol{p}_{s}^{K}\right\rangle-\left\langle\boldsymbol{m}^{K}, \boldsymbol{w}_{s}^{K}\right\rangle+\left\langle\boldsymbol{v}^{\circ}(s) \times \boldsymbol{n}^{K}, \boldsymbol{w}^{K}\right\rangle \\
& \equiv-\left\langle\boldsymbol{n}^{K}, \boldsymbol{v}_{\tau}^{K}\right\rangle-\left\langle\boldsymbol{m}^{K}, \boldsymbol{D}^{\circ} \cdot \mathbf{u}_{\tau}^{K}\right\rangle+\left\langle\boldsymbol{v}^{\circ} \times \boldsymbol{n}^{K}, \boldsymbol{w}^{K}\right\rangle \\
& \equiv-\left\langle\boldsymbol{D}^{\circ} \cdot \mathbf{n}^{K}, \boldsymbol{D}^{\circ} \cdot \mathbf{v}_{\tau}^{K}+\boldsymbol{w}^{K} \times \boldsymbol{v}^{\circ}\right\rangle-\left\langle\boldsymbol{D}^{\circ} \cdot \mathbf{m}^{K}, \boldsymbol{D}^{\circ} \cdot \mathbf{u}_{\tau}^{K}\right\rangle+\left\langle\boldsymbol{v}^{\circ} \times \boldsymbol{n}^{K}, \boldsymbol{w}^{K}\right\rangle \\
& \equiv-\left\langle\mathbf{n}^{K}, \mathbf{v}_{\tau}^{K}\right\rangle-\left\langle\mathbf{m}^{K}, \mathbf{u}_{\tau}^{K}\right\rangle \\
& \leq-c\left\{\left\|\mathbf{v}_{\tau}^{K}\right\|^{2}+\left\|\mathbf{u}_{\tau}^{K}\right\|^{2}\right\}
\end{aligned}
$$

by (2.32). Now, (8.13) and (8.14) imply that

$$
\mathbf{v}_{\tau}^{K}=\mathbf{D}^{\circ \top} \cdot \boldsymbol{p}_{s}^{K}-\mathbf{w}^{K} \times \mathbf{v}^{\circ}, \quad \mathbf{u}_{\tau}^{K}=\mathbf{D}^{\circ \top} \cdot \boldsymbol{w}_{s}^{K},
$$

and (8.3) implies (the Poincaré inequalities)

$$
\left\|\boldsymbol{p}_{s}^{K}\right\|^{2}=\sum_{k=1}^{K} k^{2} \pi^{2}\left|\boldsymbol{p}_{k}\right|^{2} \geq \pi^{2} \sum_{k=1}^{K}\left|\boldsymbol{p}_{k}\right|^{2} \geq c\left\langle\boldsymbol{p}^{K}, \rho A \boldsymbol{p}^{K}\right\rangle, \quad \text { etc. }
$$

Thus

$$
\begin{aligned}
\left\|\mathbf{v}_{\tau}^{K}\right\|^{2}+\left\|\mathbf{u}_{\tau}^{K}\right\|^{2} & \geq\left\|\boldsymbol{p}_{s}^{K}\right\|^{2}-2\left\|\boldsymbol{p}_{s}^{K}\right\|\left\|\boldsymbol{w}^{K} \times \boldsymbol{v}^{\circ}\right\|+\left\|\boldsymbol{w}^{K} \times \boldsymbol{v}^{\circ}\right\|^{2}+\left\|\boldsymbol{w}_{s}^{K}\right\|^{2} \\
& \geq(1-\eta)\left\|\boldsymbol{p}_{s}^{K}\right\|^{2}-\left(\eta^{-1}-1\right)\left\|\boldsymbol{w}^{K} \times \boldsymbol{v}^{\circ}\right\|^{2}+\left\|\boldsymbol{w}_{s}^{K}\right\|^{2} \\
& \geq c(1-\eta)\left\langle\boldsymbol{p}^{K}, \rho A \boldsymbol{p}^{K}\right\rangle-C\left\|\boldsymbol{w}^{K}\right\|^{2}+c\left\langle\boldsymbol{w}^{K}, \rho \boldsymbol{I}_{0} \cdot \boldsymbol{w}^{K}\right\rangle
\end{aligned}
$$

where $\eta \in(0,1)$ and $C$ is a bound for $v^{\circ}$, which is independent of the inertias $\rho A$ and $\rho \mathbf{I}$. Without loss of generality we can take $\rho \mathbf{I}$ to be so large that there is a positive constant $c$ such that (8.22) exceeds

$$
c\left[\left\langle\boldsymbol{p}^{K}, \rho A \boldsymbol{p}^{K}\right\rangle+\left\langle\boldsymbol{w}^{K}, \rho \boldsymbol{I}_{0} \cdot \boldsymbol{w}^{K}\right\rangle\right]
$$

because we can change the size of $\rho \mathbf{I}$ merely by having it compensate for the size of $\varepsilon$. This causes no difficulty because our asymptotic expansion will be proved to be valid 
on a finite time interval for a sufficiently small $\varepsilon$. Thus 8.19) implies that there is a (different) $c>0$ such that

$$
\frac{d}{d \tau}\left[\left\langle\boldsymbol{p}^{K}, \rho A \boldsymbol{p}^{K}\right\rangle+\left\langle\boldsymbol{w}^{K}, \rho \boldsymbol{I}_{0} \cdot \boldsymbol{w}^{K}\right\rangle\right] \leq-c\left[\left\langle\boldsymbol{p}^{K}, \rho A \boldsymbol{p}^{K}\right\rangle+\left\langle\boldsymbol{w}^{K}, \rho \boldsymbol{I}_{0} \cdot \boldsymbol{w}^{K}\right\rangle\right],
$$

so that there are positive constants $c$ and $C$ independent of $K$ such that

$$
\left\|\boldsymbol{p}^{K}(s, \tau)\right\|,\left\|\boldsymbol{w}^{K}(s, \tau)\right\| \leq C e^{-c \tau} .
$$

(It follows that $\left|\boldsymbol{p}_{s}^{K}(s, \tau)\right| \leq C K \pi e^{-c \tau},\left|\boldsymbol{w}_{s}^{K}(s, \tau)\right| \leq C K \pi e^{-c \tau}$, but these exponential bounds are not uniform. Similar nonuniform exponential bounds hold for higher $s$ derivatives of $\boldsymbol{p}^{K}$ and $\boldsymbol{w}^{K}$.)

$L^{2}$ bounds for $\boldsymbol{p}_{\tau}^{K}$ and $\boldsymbol{w}_{\tau}^{K}$. Differentiate (8.17) and (8.20) with respect to $\tau$ to get

$$
\begin{aligned}
\left\langle\rho A \boldsymbol{p}_{\tau \tau}^{K}, y_{k}\right\rangle & =-\left\langle\boldsymbol{n}_{\tau}^{K}, y_{k s}\right\rangle, \\
\left\langle\rho \boldsymbol{I}_{0} \cdot \boldsymbol{w}_{\tau \tau}^{K}, y_{k}\right\rangle & =-\left\langle\boldsymbol{m}_{\tau}^{K}, y_{k s}\right\rangle+\left\langle\boldsymbol{v}^{\circ} \times \boldsymbol{n}_{\tau}^{K}, y_{k}\right\rangle, \\
\mathbf{v}_{\tau \tau}^{K} & =\mathbf{D}^{\circ \top} \cdot \boldsymbol{p}_{s \tau}^{K}-\mathbf{D}^{\circ \top} \cdot \boldsymbol{w}_{\tau}^{K} \times \boldsymbol{v}^{\circ}, \\
\mathbf{u}_{\tau \tau}^{K} & =\mathbf{D}^{\circ \top} \cdot \boldsymbol{w}_{s \tau}^{K}, \\
\boldsymbol{n}_{\tau}^{K} & :=\boldsymbol{D}^{\circ} \cdot \hat{\mathbf{n}}_{\dot{\boldsymbol{\xi}}} \cdot \boldsymbol{\xi}_{\tau \tau}^{K}, \quad \text { etc. },
\end{aligned}
$$

where the arguments of $\hat{\mathbf{n}}_{\mathbf{v}}$, etc., are those shown in (8.16). We follow the development leading to (8.19) to obtain

$$
\begin{aligned}
& \frac{1}{2} \frac{d}{d \tau}\left[\left\langle\rho A \boldsymbol{p}_{\tau}^{K}, \boldsymbol{p}_{\tau}^{K}\right\rangle+\left\langle\rho \boldsymbol{I}_{0} \cdot \boldsymbol{w}_{\tau}^{K}, \boldsymbol{w}_{\tau}^{K}\right\rangle\right] \\
& =-\left\langle\mathbf{n}_{\tau}^{K}, \mathbf{v}_{\tau \tau}^{K}\right\rangle-\left\langle\mathbf{m}_{\tau}^{K}, \mathbf{u}_{\tau \tau}^{K}\right\rangle \leq-c\left\{\left\|\mathbf{v}_{\tau \tau}^{K}\right\|^{2}+\left\|\mathbf{u}_{\tau \tau}^{K}\right\|^{2}\right\}
\end{aligned}
$$

by (2.32). The argument supporting (8.21) yields

$$
\left\|\boldsymbol{p}_{s \tau}^{K}\right\|^{2}=\sum_{l=0}^{K} k^{2} \pi^{2}\left|\boldsymbol{p}_{k \tau}\right|^{2} \geq \pi^{2} \sum_{l=0}^{K}\left|\boldsymbol{p}_{k \tau}\right|^{2} \geq c\left\langle\boldsymbol{p}_{\tau}^{K}, \rho A \boldsymbol{p}_{\tau}^{K}\right\rangle, \quad \text { etc. }
$$

The analogs of (8.26) 3,4 yield an inequality like (8.24) with $\boldsymbol{p}^{K}, \boldsymbol{w}^{K}$ replaced with $\boldsymbol{p}_{\tau}^{K}, \boldsymbol{w}_{\tau}^{K}$, from which we obtain

$$
\left\|\boldsymbol{p}_{\tau}^{K}(s, \tau)\right\|,\left\|\boldsymbol{w}_{\tau}^{K}(s, \tau)\right\| \leq C e^{-c \tau} .
$$

From (8.19), 8.25), 8.29) we obtain

$$
\left\|\mathbf{v}_{\tau}^{K}\right\|^{2}+\left\|\mathbf{u}_{\tau}^{K}\right\|^{2} \leq C\left[\left\langle\rho A \boldsymbol{p}^{K}, \boldsymbol{p}_{\tau}^{K}\right\rangle+\left\langle\rho \boldsymbol{I}_{0} \cdot \boldsymbol{w}^{K}, \boldsymbol{w}_{\tau}^{K}\right\rangle\right] \leq C e^{-c \tau} .
$$

Conditions (8.14), 8.15), 8.25) then give

$$
\left\|\boldsymbol{p}_{s}^{K}\right\|,\left\|\boldsymbol{w}_{s}^{K}\right\| \leq C e^{-c \tau} .
$$

Since $\boldsymbol{p}^{K}(s, \tau)=\int_{0}^{s} \boldsymbol{p}_{s}^{K}(\xi, \tau) d \xi$, etc., the Cauchy-Bunyakovskiü-Schwarz inequality implies that

$$
\left|\boldsymbol{p}^{K}\right|,\left|\boldsymbol{w}^{K}\right| \leq C e^{-c \tau}
$$


Pointwise bounds for $\boldsymbol{X}^{K} \cdot \boldsymbol{n}^{K}$ and $\boldsymbol{X}^{K} \cdot \boldsymbol{m}^{K}$. Multiply (8.18) $)_{1}$ by $x_{k} /(k \pi)$ and sum the resulting equation over $k$ to get

$$
-\boldsymbol{X}^{K} \cdot \boldsymbol{n}^{K}=\sum_{k=1}^{K}\left\langle\boldsymbol{Y}^{K} \cdot \rho A \boldsymbol{p}_{\tau}^{K}, y_{k}\right\rangle \frac{x_{k}}{k \pi} .
$$

Conditions (8.29), (8.33), and the convergence of $\sum_{k=1}^{\infty} k^{-2}$ imply that

$$
\left\|\boldsymbol{X}^{K} \cdot \boldsymbol{n}^{K}\right\|^{2}=\sum_{k=1}^{K}\left|\frac{\left\langle\boldsymbol{Y}^{K} \cdot \rho A \boldsymbol{p}_{\tau}^{K}, y_{k}\right\rangle}{k \pi}\right|^{2} \leq C e^{-c \tau} .
$$

From (8.33) and (8.18) we obtain

$$
\begin{aligned}
\left\langle\boldsymbol{Y}^{K} \cdot \rho A \boldsymbol{p}_{\tau}^{K}, y_{k}\right\rangle & =\left\langle\boldsymbol{X}^{K} \cdot \boldsymbol{n}^{K}, y_{k s}\right\rangle=-\left\langle\left(\boldsymbol{X}^{K} \cdot \boldsymbol{n}^{K}\right)_{s}, y_{k}\right\rangle=-\left\langle\boldsymbol{Y}^{K} \cdot\left(\boldsymbol{X}^{K} \cdot \boldsymbol{n}^{K}\right)_{s}, y_{k}\right\rangle \\
& =\left\langle\boldsymbol{n}^{K}, y_{k s}\right\rangle=-\left\langle\boldsymbol{n}_{s}^{K}, y_{k}\right\rangle=-\left\langle\boldsymbol{Y}^{K} \cdot \boldsymbol{n}_{s}^{K}, y_{k}\right\rangle,
\end{aligned}
$$

so that

$$
\begin{gathered}
\boldsymbol{Y}^{K} \cdot \rho A \boldsymbol{p}_{\tau}^{K}=-\boldsymbol{Y}^{K} \cdot\left(\boldsymbol{X}^{K} \cdot \boldsymbol{n}^{K}\right)_{s}=-\boldsymbol{Y}^{K} \cdot \boldsymbol{n}_{s}^{K} \\
\left\|\boldsymbol{Y}^{K} \cdot\left(\boldsymbol{X}^{K} \cdot \boldsymbol{n}^{K}\right)_{s}\right\| \equiv\left\|\boldsymbol{Y}^{K} \cdot \boldsymbol{n}_{s}^{K}\right\| \leq C e^{-c \tau} .
\end{gathered}
$$

Now

$$
\begin{aligned}
\boldsymbol{Y}^{K} \cdot\left(\boldsymbol{X}^{K} \cdot \boldsymbol{n}^{K}\right)_{s} & =-\sum_{l=1}^{K}\left\langle\sum_{k=1}^{K}\left\langle\boldsymbol{n}^{K}, x_{k}\right\rangle k \pi y_{k}, y_{l}\right\rangle y_{l}=-\sum_{k=1}^{K}\left\langle\boldsymbol{n}^{K}, x_{k}\right\rangle k \pi y_{k} \\
& =\partial_{s} \sum_{k=1}^{K}\left\langle\boldsymbol{n}^{K}, x_{k}\right\rangle x_{k}=\left(\boldsymbol{X}^{K} \cdot \boldsymbol{n}^{K}\right)_{s} .
\end{aligned}
$$

Thus (8.36) implies that

$$
\left\|\left(\boldsymbol{X}^{K} \cdot \boldsymbol{n}^{K}\right)_{s}\right\| \leq C e^{-c \tau}
$$

Since

$$
\mathbf{e}_{j} \cdot\left(\boldsymbol{X}^{K} \cdot \boldsymbol{n}^{K}\right)(s, \tau)-\mathbf{e}_{j} \cdot \int_{0}^{1}\left(\boldsymbol{X}^{K} \cdot \boldsymbol{n}^{K}\right)(\xi, \tau) d \xi=\mathbf{e}_{j} \cdot \int_{\sigma_{j}(\tau)}^{s}\left(\boldsymbol{X}^{K} \cdot \boldsymbol{n}^{K}\right)_{s}(\xi, \tau) d \xi
$$

where $\sigma_{j}(\tau)$ is the value of $s$ at which the left-hand side of this equation vanishes, it follows from (8.34) and (8.38) that

$$
\left|\left(\boldsymbol{X}^{K} \cdot \boldsymbol{n}^{K}\right)(s, \tau)\right| \leq C e^{-c \tau} .
$$

The substitution of (8.29) and (8.40) into (8.17) 2 and the argument leading to (8.39) immediately leads to

$$
\left|\left(\boldsymbol{X}^{K} \cdot \boldsymbol{m}^{K}\right)(s, \tau)\right| \leq C e^{-c \tau} .
$$

Pointwise bounds for $\boldsymbol{v}_{\tau}^{K}, \boldsymbol{u}_{\tau}^{K}, \boldsymbol{p}_{s}^{K}, \boldsymbol{w}_{s}^{K}$. By definition and by the Mean-Value Theorem,

$$
\begin{aligned}
\boldsymbol{X}^{K} \cdot\left\{\begin{array}{c}
\boldsymbol{n}^{K} \\
\boldsymbol{m}^{K}
\end{array}\right\} & =\boldsymbol{X}^{K} \cdot \boldsymbol{D}^{\circ} \cdot\left[\left\{\begin{array}{c}
\hat{\mathbf{n}} \\
\hat{\mathbf{m}}
\end{array}\right\}\left(\boldsymbol{\xi}_{0}(\cdot, 0), \boldsymbol{\xi}_{0 t}(\cdot, 0)+\boldsymbol{\xi}_{\tau}^{K}, \cdot\right)-\left\{\begin{array}{c}
\hat{\mathbf{n}} \\
\hat{\mathbf{m}}
\end{array}\right\}\left(\boldsymbol{\xi}_{0}(\cdot, 0), \boldsymbol{\xi}_{0 t}(\cdot, 0), \cdot\right)\right] \\
& =\boldsymbol{X}^{K} \cdot \boldsymbol{D}^{\circ} \cdot \int_{0}^{1}\left\{\begin{array}{c}
\hat{\mathbf{n}}_{\dot{\boldsymbol{\xi}}} \\
\hat{\mathbf{m}}_{\boldsymbol{\xi}}
\end{array}\right\} d \alpha \cdot \boldsymbol{\xi}_{\tau}^{K}
\end{aligned}
$$


where the arguments of the derivatives of $\hat{\mathbf{n}}$ and $\hat{\mathbf{m}}$ are $\left(\boldsymbol{\xi}_{0}(\cdot, 0), \boldsymbol{\xi}_{0 t}(\cdot, 0)+\alpha \boldsymbol{\xi}_{\tau}^{K}, \cdot\right)$. Condition (8.14) implies that

$$
\boldsymbol{v}_{\tau}^{K}=\boldsymbol{p}_{s}^{K}=\sum_{k=0}^{K} k \pi \boldsymbol{p}_{k} x_{k}=\boldsymbol{X}^{K} \cdot \boldsymbol{v}_{\tau}^{K}=\boldsymbol{X}^{K} \cdot \boldsymbol{p}_{s}^{K}, \quad \boldsymbol{w}_{s}^{K}=\boldsymbol{X}^{K} \cdot \boldsymbol{w}_{s}^{K},
$$

so that (8.14) and (8.15) imply that

$$
\mathbf{v}_{\tau}^{K}=\mathbf{D}^{\circ \top} \cdot\left[\boldsymbol{X}^{K} \cdot \boldsymbol{p}_{s}^{K}-\boldsymbol{w}^{K} \times \boldsymbol{v}^{\circ}\right], \quad \mathbf{u}_{\tau}^{K}=\mathbf{D}^{\circ \boldsymbol{T}} \cdot \boldsymbol{X}^{K} \cdot \boldsymbol{w}_{s}^{K} .
$$

We substitute (8.44) into the right-hand side of (8.42) and observe that the self-adjointness of the projection $\boldsymbol{X}^{K}$ and condition (2.32) ensure that the matrix of coefficients of this right-hand side with respect to $\left(\boldsymbol{p}_{s}^{K}, \boldsymbol{w}_{s}^{K}\right) \equiv\left(\boldsymbol{v}_{\tau}^{K}, \boldsymbol{u}_{\tau}^{K}\right)$ is uniformly positive-definite, so that this system can be solved for the visible $\left(\boldsymbol{p}_{s}^{K}, \boldsymbol{w}_{s}^{K}\right)$ in terms of the left-hand side of (8.42) and (the troublesome term)

$$
\boldsymbol{X}^{K} \cdot \boldsymbol{D}^{\circ} \cdot \int_{0}^{1}\left\{\begin{array}{c}
\hat{\mathbf{n}}_{\dot{\mathrm{v}}} \\
\hat{\mathbf{m}}_{\dot{\mathrm{v}}}
\end{array}\right\} d \alpha \cdot \mathbf{D}^{\circ \mathrm{T}} \cdot \boldsymbol{w}^{K} \times \boldsymbol{v}^{\circ} .
$$

We now complement (2.32), which gives a sort of lower bound on the derivatives of the constitutive functions, with a mild sort of upper bound: There is a real-valued continuous function $h$ on $\mathbb{E}^{3} \times \mathbb{E}^{3}$ such that

$$
\left|\boldsymbol{X}^{K} \cdot \boldsymbol{D}^{\circ} \cdot \hat{\mathbf{n}}_{\dot{\mathbf{v}}}\right|,\left|\boldsymbol{X}^{K} \cdot \boldsymbol{D}^{\circ} \cdot \hat{\mathbf{n}}_{\mathbf{u}}\right|,\left|\boldsymbol{X}^{K} \cdot \boldsymbol{D}^{\circ} \cdot \hat{\mathbf{m}}_{\dot{\mathbf{v}}}\right|,\left|\boldsymbol{X}^{K} \cdot \boldsymbol{D}^{\circ} \cdot \hat{\mathbf{m}}_{\dot{\mathbf{u}}}\right| \leq h\left(\boldsymbol{X}^{K} \cdot \boldsymbol{D}^{\circ} \cdot \hat{\mathbf{n}}, \boldsymbol{X}^{K} \cdot \boldsymbol{D}^{\circ} \cdot \hat{\mathbf{m}}\right) .
$$

This condition, together with the uniform exponential decay (8.40) and (8.41) of the left-hand side of (8.42) and the uniform exponential decay (8.25) of $\boldsymbol{w}^{K}$, implies that of (8.45). The solvability of (8.42) for $\left(\boldsymbol{p}_{s}^{K}, \boldsymbol{w}_{s}^{K}\right)$, these results on exponential decay, and (8.44) immediately give

$$
\left|\boldsymbol{p}_{s}^{K}(s, \tau)\right|,\left|\boldsymbol{w}_{s}^{K}(s, \tau)\right|,\left|\mathbf{v}_{\tau}^{K}(s, \tau)\right|,\left|\mathbf{u}_{\tau}^{K}(s, \tau)\right| \leq C e^{-c \tau} .
$$

Consequently all the derivatives of $\hat{\mathbf{n}}$ and $\hat{\mathbf{m}}$ evaluated at the arguments shown in (8.42) are bounded.

$L^{2}$ bounds for $\boldsymbol{p}_{s s}^{K}, \boldsymbol{w}_{s s}^{K}, \mathbf{v}_{s \tau}^{K}, \mathbf{u}_{s \tau}^{K}$. Write (8.17) as

$$
\begin{aligned}
\left\langle\rho A \boldsymbol{p}_{\tau}^{K}, y_{k}\right\rangle & =\left\langle\boldsymbol{n}_{s}^{K}, y_{k}\right\rangle \equiv\left\langle\boldsymbol{D}^{\circ} \cdot\left[\mathbf{n}_{\dot{\boldsymbol{\xi}}}^{K} \cdot \boldsymbol{\xi}_{s \tau}^{K}+\mathbf{n}_{\bar{s}}^{K}\right]+\boldsymbol{u}^{\circ} \times \boldsymbol{D}^{\circ} \cdot \mathbf{n}^{K}, y_{k}\right\rangle, \\
\left\langle\rho \boldsymbol{I}_{0} \cdot \boldsymbol{w}_{\tau}^{K}, y_{k}\right\rangle & =\left\langle\boldsymbol{m}_{s}^{K}, y_{k}\right\rangle+\left\langle\boldsymbol{v}^{\circ} \times \boldsymbol{n}^{K}, y_{k}\right\rangle \\
& \equiv\left\langle\boldsymbol{D}^{\circ} \cdot\left[\mathbf{m}_{\dot{\boldsymbol{\xi}}}^{K} \cdot \boldsymbol{\xi}_{s \tau}^{K}+\mathbf{m}_{\bar{s}}^{K}\right]+\boldsymbol{u}^{\circ} \times \boldsymbol{D}^{\circ} \cdot \mathbf{m}^{K}, y_{k}\right\rangle+\left\langle\boldsymbol{v}^{\circ} \times \boldsymbol{n}^{K}, y_{k}\right\rangle .
\end{aligned}
$$

Here the subscript $\bar{s}$ denotes the derivatives of $\hat{\mathbf{n}}$ and $\hat{\mathbf{m}}$ with respect to the $s$ appearing in $\mathbf{v}^{\circ}(s), \mathbf{u}^{\circ}(s), \mathbf{v}_{0 t}(s, 0), \mathbf{u}_{0 t}(s, 0)$, and the last arguments of $\hat{\mathbf{n}}$ and $\hat{\mathbf{m}}$.

Multiply these equations respectively by $k^{2} \pi^{2} \boldsymbol{p}_{k}$ and $k^{2} \pi^{2} \boldsymbol{w}_{k}$, use (8.2), sum the products over $k$, and add the two sums to obtain

$$
\begin{aligned}
\left\langle\rho A \boldsymbol{p}_{\tau}^{K}, \boldsymbol{p}_{s s}^{K}\right\rangle+\left\langle\rho \boldsymbol{I}_{0} \cdot \boldsymbol{w}_{\tau}^{K}, \boldsymbol{w}_{s s}^{K}\right\rangle= & \left\langle\boldsymbol{D}^{\circ} \cdot\left[\mathbf{n}_{\dot{\boldsymbol{\xi}}}^{K} \cdot \boldsymbol{\xi}_{s \tau}^{K}+\mathbf{n}_{\bar{s}}^{K}\right]+\boldsymbol{u}^{\circ} \times \boldsymbol{D}^{\circ} \cdot \mathbf{n}^{K}, \boldsymbol{p}_{s s}^{K}\right\rangle \\
& +\left\langle\boldsymbol{D}^{\circ} \cdot\left[\mathbf{m}_{\dot{\boldsymbol{\xi}}}^{K} \cdot \boldsymbol{\xi}_{s \tau}^{K}+\mathbf{m}_{\bar{s}}^{K}\right]+\boldsymbol{u}^{\circ} \times \boldsymbol{D}^{\circ} \cdot \boldsymbol{m}^{K}, \boldsymbol{w}_{s s}^{K}\right\rangle \\
& +\left\langle\boldsymbol{v}^{\circ} \times \boldsymbol{n}^{K}, \boldsymbol{w}_{s s}^{K}\right\rangle
\end{aligned}
$$


where

$$
\begin{aligned}
\boldsymbol{p}_{s s}^{K} & \equiv \boldsymbol{v}_{s \tau}^{K}=\boldsymbol{D}^{\circ} \cdot \mathbf{v}_{s \tau}^{K}+\boldsymbol{u}^{\circ} \times \boldsymbol{D}^{\circ} \cdot \mathbf{v}_{\tau}^{K}+\boldsymbol{w}_{s}^{K} \times \boldsymbol{v}^{\circ}+\boldsymbol{w}^{K} \times \boldsymbol{v}_{0 s}(\cdot, 0), \\
\boldsymbol{w}_{s s}^{K} & =\boldsymbol{D}^{\circ} \cdot \mathbf{u}_{s \tau}^{K}+\boldsymbol{u}^{\circ} \times \boldsymbol{D}^{\circ} \cdot \mathbf{u}_{\tau}^{K}
\end{aligned}
$$

by (8.14). Condition (2.32) implies that the integrand of the right-hand side of (8.49) is

$$
\mathbf{v}_{s \tau}^{K} \cdot \mathbf{n}_{\dot{\mathbf{v}}}^{K} \cdot \mathbf{v}_{s \tau}^{K}+\mathbf{v}_{s \tau}^{K} \cdot \mathbf{n}_{\dot{\mathbf{v}}}^{K} \cdot \mathbf{u}_{s \tau}^{K}+\mathbf{u}_{s \tau}^{K} \cdot \mathbf{m}_{\dot{\mathbf{v}}}^{K} \cdot \mathbf{v}_{s \tau}^{K}+\mathbf{u}_{s \tau}^{K} \cdot \mathbf{m}_{\dot{\mathbf{v}}}^{K} \cdot \mathbf{u}_{s \tau}^{K}+f \geq c\left(\left|\mathbf{v}_{s \tau}^{K}\right|^{2}+\left|\mathbf{u}_{s \tau}^{K}\right|^{2}\right)+f
$$

where

$$
\begin{aligned}
f:= & {\left[\boldsymbol{D}^{\circ} \cdot \mathbf{n}_{\bar{s}}^{K}+\boldsymbol{u}^{\circ} \times \boldsymbol{D}^{\circ} \cdot \mathbf{n}^{K}\right] \cdot \boldsymbol{D}^{\circ} \cdot \mathbf{v}_{s \tau}^{K}+\left[\boldsymbol{D}^{\circ} \cdot \mathbf{m}_{\bar{s}}^{K}+\boldsymbol{u}^{\circ} \times \boldsymbol{D}^{\circ} \cdot \mathbf{m}^{K}\right] \cdot \boldsymbol{D}^{\circ} \cdot \mathbf{u}_{s \tau}^{K} } \\
& +\boldsymbol{D}^{\circ} \cdot\left[\mathbf{n}_{\dot{\xi}}^{K} \cdot \boldsymbol{\xi}_{s \tau}^{K}+\mathbf{n}_{\bar{s}}^{K}+\boldsymbol{u}^{\circ} \times \boldsymbol{D}^{\circ} \cdot \mathbf{n}^{K}\right] \\
& \cdot\left[\boldsymbol{u}^{\circ} \times \boldsymbol{D}^{\circ}(s) \cdot \mathbf{v}_{\tau}^{K}+\boldsymbol{w}_{s}^{K} \times \boldsymbol{v}^{\circ}+\boldsymbol{w}^{K} \times \boldsymbol{v}_{0 s}(\cdot, 0)\right] \\
& +\boldsymbol{D}^{\circ} \cdot\left[\mathbf{m}_{\dot{\boldsymbol{\xi}}}^{K} \cdot \boldsymbol{\xi}_{s \tau}^{K}+\mathbf{m}_{\bar{s}}^{K}+\boldsymbol{u}^{\circ} \times \boldsymbol{D}^{\circ} \cdot \mathbf{m}^{K}\right] \cdot\left[\boldsymbol{u}^{\circ} \times \boldsymbol{D}^{\circ} \cdot \mathbf{u}_{\tau}^{K}\right]+\left[\boldsymbol{v}^{\circ} \times \boldsymbol{n}^{K}\right] \cdot \boldsymbol{w}_{s s}^{K}
\end{aligned}
$$

The function $f$ is affine in $\boldsymbol{\xi}_{s \tau}^{K}$ with coefficients depending on $\boldsymbol{\xi}_{\tau}^{K}$ and $\mathbf{u}_{\tau}^{K}$ that are exponentially decaying by virtue of (8.42), of analogous applications of the Mean-Value Theorem to $\mathbf{n}_{\bar{s}}^{K}$ and $\mathbf{m}_{\bar{s}}^{K}$, and of (8.47). Equivalently, (8.50) shows that the rightmost term of (8.51) is a positive-definite (nonhomogeneous) quadratic form in $\boldsymbol{p}_{s s}^{K}$ and $\boldsymbol{w}_{s s}^{K}$. Setting $z:=\left\|\boldsymbol{p}_{s s}^{K}\right\|+\left\|\boldsymbol{w}_{s s}^{K}\right\|$ we accordingly obtain from (8.49)- (8.52) that $z^{2}-C z e^{-c \tau} \leq$ $C e^{-c \tau}$, whence

$$
\left\|\boldsymbol{p}_{s s}^{K}\right\|,\left\|\boldsymbol{w}_{s s}^{K}\right\|,\left\|\mathbf{v}_{s \tau}^{K}\right\|,\left\|\mathbf{u}_{s \tau}^{K}\right\| \leq C e^{-c \tau}
$$

(These estimates imply (8.47).)

Pointwise bounds for $\boldsymbol{p}_{\tau}^{K}, \boldsymbol{w}_{\tau}^{K}$. System (8.26) implies that $\boldsymbol{p}_{\tau}, \boldsymbol{w}_{\tau}$ satisfy (the linear system)

$$
\begin{aligned}
\left\langle\rho A \boldsymbol{p}_{\tau \tau \tau}^{K}, y_{k}\right\rangle & =-\left\langle\boldsymbol{n}_{\tau \tau}^{K}, x_{k s}\right\rangle, \\
\left\langle\rho \boldsymbol{I}_{0} \cdot \boldsymbol{w}_{\tau \tau \tau}^{K}, y_{k}\right\rangle & =-\left\langle\boldsymbol{m}_{\tau \tau}^{K}, y_{k s}\right\rangle+\left\langle\boldsymbol{v}^{\circ} \times \boldsymbol{n}_{\tau \tau}^{K}, y_{k}\right\rangle, \\
\mathbf{v}_{\tau \tau}^{K} & =\mathbf{D}^{\circ \boldsymbol{\top}} \cdot \boldsymbol{p}_{s \tau}^{K}-\mathbf{D}^{\circ \mathrm{T}} \cdot \boldsymbol{w}_{\tau}^{K} \times \boldsymbol{v}^{\circ}, \quad \mathbf{u}_{\tau \tau}^{K}=\mathbf{D}^{\circ \boldsymbol{\top}} \cdot \boldsymbol{w}_{s \tau}^{K}, \\
\mathbf{v}_{\tau \tau \tau}^{K} & =\mathbf{D}^{\circ \boldsymbol{\top}} \cdot \boldsymbol{p}_{s \tau \tau}^{K}-\mathbf{D}^{\circ \top} \cdot \boldsymbol{w}_{\tau \tau}^{K} \times \boldsymbol{v}^{\circ}, \quad \mathbf{u}_{\tau \tau \tau}^{K}=\mathbf{D}^{\circ \boldsymbol{\top}} \cdot \boldsymbol{w}_{s \tau \tau}^{K}, \\
\boldsymbol{n}_{\tau}^{K} & =\boldsymbol{D}^{\circ} \cdot \hat{\mathbf{n}}_{\dot{\boldsymbol{\xi}}} \cdot \boldsymbol{\xi}_{\tau \tau}^{K}, \quad \boldsymbol{n}_{\tau \tau}^{K}=\boldsymbol{D}^{\circ} \cdot\left[\hat{\mathbf{n}}_{\dot{\boldsymbol{\xi}}} \cdot \boldsymbol{\xi}_{\tau \tau \tau}^{K}+\hat{\mathbf{n}}_{\dot{\boldsymbol{\xi}} \boldsymbol{\xi}}: \boldsymbol{\xi}_{\tau \tau}^{K} \boldsymbol{\xi}_{\tau \tau}^{K}\right], \quad \text { etc. },
\end{aligned}
$$

where the arguments of $\hat{\mathbf{n}}_{\dot{\xi}}$, etc., are those shown in (8.16). We imitate the development following (8.26). We multiply (8.54) ${ }_{1,2}$ respectively by $p_{k \tau \tau}$ and $w_{k \tau \tau}$, sum the resulting 
equations over $k$, and add the sums to obtain

$$
\begin{aligned}
\frac{1}{2} & \frac{d}{d \tau}\left[\left\langle\rho A \boldsymbol{p}_{\tau \tau}^{K}, \boldsymbol{p}_{\tau \tau}^{K}\right\rangle+\left\langle\rho \boldsymbol{I}_{0} \cdot \boldsymbol{w}_{\tau \tau}^{K}, \boldsymbol{w}_{\tau \tau}^{K}\right\rangle\right] \\
\equiv & -\left\langle\boldsymbol{n}_{\tau \tau}^{K}, \boldsymbol{p}_{s \tau \tau}^{K}\right\rangle-\left\langle\boldsymbol{m}_{\tau \tau}^{K}, \boldsymbol{w}_{s \tau \tau}^{K}\right\rangle+\left\langle\boldsymbol{v}^{\circ} \times \boldsymbol{n}_{\tau \tau}^{K}, \boldsymbol{w}_{\tau \tau}^{K}\right\rangle \\
\equiv & -\left\langle\boldsymbol{D}^{\circ} \cdot\left[\hat{\mathbf{n}}_{\dot{\xi}} \cdot \boldsymbol{\xi}_{\tau \tau \tau}^{K}+\hat{\mathbf{n}}_{\dot{\xi} \dot{\xi}}: \boldsymbol{\xi}_{\tau \tau}^{K} \boldsymbol{\xi}_{\tau \tau}^{K}\right], \boldsymbol{D}^{\circ} \cdot \mathbf{v}_{\tau \tau \tau}^{K}+\boldsymbol{w}_{\tau \tau}^{K} \times \boldsymbol{v}^{\circ}\right\rangle \\
& -\left\langle\boldsymbol{D}^{\circ} \cdot\left[\hat{\mathbf{m}}_{\dot{\xi}} \cdot \boldsymbol{\xi}_{\tau \tau \tau}^{K}+\hat{\mathbf{m}}_{\dot{\xi} \dot{\xi}}: \boldsymbol{\xi}_{\tau \tau}^{K} \boldsymbol{\xi}_{\tau \tau}^{K}\right], \boldsymbol{D}^{\circ} \cdot \mathbf{u}_{\tau \tau \tau}^{K}\right\rangle+\left\langle\boldsymbol{v}^{\circ} \times \boldsymbol{n}_{\tau \tau}, \boldsymbol{w}_{\tau \tau}^{K}\right\rangle \\
\equiv & -\left\langle\hat{\mathbf{n}}_{\dot{\xi}} \cdot \boldsymbol{\xi}_{\tau \tau \tau}^{K}, \mathbf{v}_{\tau \tau \tau}^{K}\right\rangle-\left\langle\hat{\mathbf{m}}_{\dot{\xi}}^{K} \cdot \boldsymbol{\xi}_{\tau \tau \tau}^{K}, \mathbf{u}_{\tau \tau \tau}^{K}\right\rangle+\left\langle\hat{\mathbf{n}}_{\dot{\xi} \dot{\xi}}: \boldsymbol{\xi}_{\tau \tau}^{K} \boldsymbol{\xi}_{\tau \tau}^{K}, \mathbf{v}_{\tau \tau \tau}^{K}\right\rangle+\left\langle\hat{\mathbf{m}}_{\dot{\xi} \dot{\xi}}: \boldsymbol{\xi}_{\tau \tau}^{K} \boldsymbol{\xi}_{\tau \tau}^{K}, \mathbf{u}_{\tau \tau \tau}^{K}\right\rangle \\
\leq & -c\left\|\boldsymbol{\xi}_{\tau \tau \tau}^{K}\right\|^{2}+\left\langle\hat{\mathbf{n}}_{\dot{\xi} \dot{\xi}}: \boldsymbol{\xi}_{\tau \tau}^{K} \boldsymbol{\xi}_{\tau \tau}^{K}, \mathbf{v}_{\tau \tau \tau}^{K}\right\rangle+\left\langle\hat{\mathbf{m}}_{\dot{\xi} \dot{\xi}}: \boldsymbol{\xi}_{\tau \tau}^{K} \boldsymbol{\xi}_{\tau \tau}^{K}, \mathbf{u}_{\tau \tau \tau}^{K}\right\rangle \\
\leq & -c\left\|\boldsymbol{\xi}_{\tau \tau \tau}^{K}\right\|^{2}+C\left\|\boldsymbol{\xi}_{\tau \tau}^{K}\right\|^{2}\left\|\boldsymbol{\xi}_{\tau \tau \tau}^{K}\right\| .
\end{aligned}
$$

Set

$$
g:=\left\langle\rho A \boldsymbol{p}_{\tau \tau}^{K}, \boldsymbol{p}_{\tau \tau}^{K}\right\rangle+\left\langle\rho \boldsymbol{I}_{0} \cdot \boldsymbol{w}_{\tau \tau}^{K}, \boldsymbol{w}_{\tau \tau}^{K}\right\rangle .
$$

Since (8.27) and (8.29) imply that $\left\|\boldsymbol{\xi}_{\tau \tau}^{K}\right\|^{2} \leq C e^{-c \tau} \sqrt{g}$, inequality (8.55) yields

$$
g_{\tau} \leq-c\left\|\boldsymbol{\xi}_{\tau \tau \tau}^{K}\right\|^{2}+C e^{-c \tau} \sqrt{g}\left\|\boldsymbol{\xi}_{\tau \tau \tau}^{K}\right\| \leq-c\left\|\boldsymbol{\xi}_{\tau \tau \tau}^{K}\right\|^{2}+C e^{-c \tau} g
$$

(in consonance with the convention on constants). As in (8.22), equations (8.54) imply that

$$
\left\|\boldsymbol{\xi}_{\tau \tau \tau}^{K}\right\|^{2}=\left\|\boldsymbol{q}_{s \tau}^{K}-\boldsymbol{w}_{\tau \tau} \times \boldsymbol{v}^{\circ}\right\|^{2}+\left\|\boldsymbol{w}_{s \tau \tau}\right\|^{2} \geq c g
$$

for sufficiently large $\rho \mathbf{I}$. Thus $g_{\tau} \leq-c g+C e^{-c \tau} g$, so that

$$
\left\|\boldsymbol{p}_{\tau \tau}^{K}\right\| \leq C^{-c \tau}, \quad\left\|\boldsymbol{w}_{\tau \tau}^{K}\right\| \leq C^{-c \tau}
$$

From (8.27), (8.29), 8.59), we obtain

$$
c\left\|\boldsymbol{\xi}_{\tau \tau}^{K}\right\|^{2} \leq\left\langle\mathbf{n}_{\tau}^{K}, \mathbf{v}_{\tau \tau}^{K}\right\rangle+\left\langle\mathbf{m}_{\tau}^{K}, \mathbf{u}_{\tau \tau}^{K}\right\rangle=\left\langle\rho A \boldsymbol{p}_{\tau}^{K}, \boldsymbol{p}_{\tau \tau}^{K}\right\rangle+\left\langle\rho \boldsymbol{I}_{0} \cdot \boldsymbol{w}_{\tau}^{K}, \boldsymbol{w}_{\tau \tau}^{K}\right\rangle \leq C e^{-c \tau},
$$

so (8.29) and (8.54) imply that

$$
\left\|\boldsymbol{\xi}_{\tau \tau}^{K}\right\|,\left\|\boldsymbol{p}_{s \tau}^{K}\right\|,\left\|\boldsymbol{w}_{s \tau}^{K}\right\| \leq C^{-c \tau} \quad \Longrightarrow \quad\left|\boldsymbol{p}_{\tau}^{K}\right|,\left|\boldsymbol{w}_{\tau}^{K}\right| \leq C^{-c \tau}
$$

$L^{2}$ bounds for $\boldsymbol{p}_{\tau \tau \tau}^{K}$ and $\boldsymbol{w}_{\tau \tau \tau}^{K}$. We follow the development beginning with (8.54):

$$
\begin{aligned}
\left\langle\rho A \boldsymbol{p}_{\tau \tau \tau \tau}^{K}, y_{k}\right\rangle & =-\left\langle\boldsymbol{n}_{\tau \tau \tau}^{K}, x_{k s}\right\rangle, \\
\left\langle\rho \boldsymbol{I}_{0} \cdot \boldsymbol{w}_{\tau \tau \tau \tau}^{K}, y_{k}\right\rangle & =-\left\langle\boldsymbol{m}_{\tau \tau \tau}^{K}, y_{k s}\right\rangle+\left\langle\boldsymbol{v}^{\circ} \times \boldsymbol{n}_{\tau \tau \tau}^{K}, y_{k}\right\rangle, \\
\mathbf{v}_{\tau \tau \tau \tau}^{K} & =\mathbf{D}^{\circ \boldsymbol{T}} \cdot \boldsymbol{p}_{s \tau \tau \tau}^{K}-\mathbf{D}^{\circ \top} \cdot \boldsymbol{w}_{\tau \tau \tau}^{K} \times \boldsymbol{v}^{\circ}, \quad \mathbf{u}_{\tau \tau \tau \tau}^{K}=\mathbf{D}^{\circ \top} \cdot \boldsymbol{w}_{s \tau \tau \tau}^{K}, \\
\boldsymbol{n}_{\tau \tau \tau}^{K} & =\boldsymbol{D}^{\circ} \cdot\left[\hat{\mathbf{n}}_{\dot{\xi}} \cdot \boldsymbol{\xi}_{\tau \tau \tau \tau}^{K}+3 \hat{\mathbf{n}}_{\dot{\xi} \dot{\xi}}: \boldsymbol{\xi}_{\tau \tau}^{K} \boldsymbol{\xi}_{\tau \tau \tau}^{K}+\hat{\mathbf{n}}_{\dot{\xi} \dot{\xi} \dot{\xi}} \therefore \boldsymbol{\xi}_{\tau \tau}^{K} \boldsymbol{\xi}_{\tau \tau}^{K} \boldsymbol{\xi}_{\tau \tau}^{K}\right], \quad \text { etc. },
\end{aligned}
$$

where the arguments of $\hat{\mathbf{n}}_{\dot{\xi}}$, etc., are those shown in (8.16). We imitate the development following (8.26). We multiply (8.62) ${ }_{1,2}$ respectively by $p_{k \tau \tau \tau}$ and $w_{k \tau \tau \tau}$, sum the resulting 
equations over $k$, and add the sums to obtain

$$
\begin{aligned}
& \frac{1}{2} \frac{d}{d \tau}\left[\left\langle\rho A \boldsymbol{p}_{\tau \tau \tau}^{K}, \boldsymbol{p}_{\tau \tau \tau}^{K}\right\rangle+\left\langle\rho \boldsymbol{I}_{0} \cdot \boldsymbol{w}_{\tau \tau \tau}^{K}, \boldsymbol{w}_{\tau \tau \tau}^{K}\right\rangle\right] \\
& \equiv-\left\langle\boldsymbol{n}_{\tau \tau \tau}^{K}, \boldsymbol{p}_{s \tau \tau \tau}^{K}\right\rangle-\left\langle\boldsymbol{m}_{\tau \tau \tau}^{K}, \boldsymbol{w}_{s \tau \tau \tau}^{K}\right\rangle+\left\langle\boldsymbol{v}^{\circ} \times \boldsymbol{n}_{\tau \tau \tau}^{K}, \boldsymbol{w}_{\tau \tau \tau}^{K}\right\rangle \\
& \equiv-\left\langle\boldsymbol{D}^{\circ} \cdot\left[\hat{\mathbf{n}}_{\dot{\boldsymbol{\xi}}} \cdot \boldsymbol{\xi}_{\tau \tau \tau \tau}^{K}+3 \hat{\mathbf{n}}_{\dot{\boldsymbol{\xi}} \dot{\xi}}: \boldsymbol{\xi}_{\tau \tau}^{K} \boldsymbol{\xi}_{\tau \tau \tau}^{K}+\hat{\mathbf{n}}_{\dot{\boldsymbol{\xi}} \dot{\xi} \dot{\xi}} \therefore \boldsymbol{\xi}_{\tau \tau}^{K} \boldsymbol{\xi}_{\tau \tau}^{K} \boldsymbol{\xi}_{\tau \tau}^{K}\right], \boldsymbol{D}^{\circ} \cdot \mathbf{v}_{\tau \tau \tau \tau}^{K}+\boldsymbol{w}_{\tau \tau \tau}^{K} \times \boldsymbol{v}^{\circ}\right\rangle \\
& -\left\langle\boldsymbol{D}^{\circ} \cdot\left[\hat{\mathbf{m}}_{\dot{\boldsymbol{\xi}}} \cdot \boldsymbol{\xi}_{\tau \tau \tau \tau}^{K}+3 \hat{\mathbf{m}}_{\dot{\boldsymbol{\xi} \dot{\xi}}}: \boldsymbol{\xi}_{\tau \tau}^{K} \boldsymbol{\xi}_{\tau \tau \tau}^{K}+\hat{\mathbf{m}}_{\dot{\boldsymbol{\xi} \dot{\xi} \boldsymbol{\xi}}} \therefore \boldsymbol{\xi}_{\tau \tau}^{K} \boldsymbol{\xi}_{\tau \tau}^{K} \boldsymbol{\xi}_{\tau \tau}^{K}\right], \boldsymbol{D}^{\circ} \cdot \mathbf{u}_{\tau \tau \tau \tau}^{K}\right\rangle \\
& +\left\langle\boldsymbol{v}^{\circ} \times \boldsymbol{n}_{\tau \tau \tau}, \boldsymbol{w}_{\tau \tau \tau}^{K}\right\rangle \\
& \equiv-\left\langle\hat{\mathbf{n}}_{\dot{\boldsymbol{\xi}}} \cdot \boldsymbol{\xi}_{\tau \tau \tau \tau}^{K}, \mathbf{v}_{\tau \tau \tau \tau}^{K}\right\rangle-\left\langle\hat{\mathbf{m}}_{\dot{\boldsymbol{\xi}}} \cdot \boldsymbol{\xi}_{\tau \tau \tau \tau}^{K}, \mathbf{u}_{\tau \tau \tau \tau}^{K}\right\rangle+3\left\langle\hat{\mathbf{n}}_{\dot{\boldsymbol{\xi}} \boldsymbol{\xi}}: \boldsymbol{\xi}_{\tau \tau}^{K} \boldsymbol{\xi}_{\tau \tau \tau}^{K}, \mathbf{v}_{\tau \tau \tau \tau}^{K}\right\rangle \\
& +3\left\langle\hat{\mathbf{m}}_{\dot{\xi} \dot{\xi}}: \boldsymbol{\xi}_{\tau \tau}^{K} \boldsymbol{\xi}_{\tau \tau \tau}^{K}, \mathbf{u}_{\tau \tau \tau \tau}^{K}\right\rangle+\left\langle\hat{\mathbf{n}}_{\dot{\xi} \dot{\xi} \dot{\xi}} \therefore \boldsymbol{\xi}_{\tau \tau}^{K} \boldsymbol{\xi}_{\tau \tau}^{K} \boldsymbol{\xi}_{\tau \tau}^{K}, \mathbf{v}_{\tau \tau \tau \tau}^{K}\right\rangle+\left\langle\hat{\mathbf{m}}_{\dot{\xi} \dot{\xi} \dot{\xi}} \therefore \boldsymbol{\xi}_{\tau \tau}^{K} \boldsymbol{\xi}_{\tau \tau}^{K} \boldsymbol{\xi}_{\tau \tau}^{K}, \mathbf{u}_{\tau \tau \tau \tau}^{K}\right\rangle \\
& \leq-c\left\|\boldsymbol{\xi}_{\tau \tau \tau \tau}^{K}\right\|^{2}+C\left\|\boldsymbol{\xi}_{\tau \tau}^{K}\right\|\left\|\boldsymbol{\xi}_{\tau \tau \tau}^{K}\right\|\left\|\boldsymbol{\xi}_{\tau \tau \tau \tau}^{K}\right\|+C\left\|\boldsymbol{\xi}_{\tau \tau}^{K}\right\|^{3}\left\|\boldsymbol{\xi}_{\tau \tau \tau \tau}^{K}\right\| .
\end{aligned}
$$

Set

$$
h:=\left\langle\rho A \boldsymbol{p}_{\tau \tau \tau}^{K}, \boldsymbol{p}_{\tau \tau \tau}^{K}\right\rangle+\left\langle\rho \boldsymbol{I}_{0} \cdot \boldsymbol{w}_{\tau \tau \tau}^{K}, \boldsymbol{w}_{\tau \tau \tau}^{K}\right\rangle .
$$

Relations (8.55), (8.59), (8.60) imply that

$$
c\left\|\boldsymbol{\xi}_{\tau \tau \tau}^{K}\right\|^{2}-C e^{-c \tau} \leq c\left\|\boldsymbol{\xi}_{\tau \tau \tau}^{K}\right\|^{2}-C e^{-c \tau}\left\|\boldsymbol{\xi}_{\tau \tau \tau}^{K}\right\| \leq C e^{-c \tau} \sqrt{h} \leq C e^{-c \tau}+h .
$$

Inequalities (8.60), (8.63), (8.63) yield

$$
h_{\tau} \leq-c\left\|\boldsymbol{\xi}_{\tau \tau \tau \tau}^{K}\right\|^{2}+C \varepsilon^{-c \tau}[\sqrt{h}+1]\left\|\boldsymbol{\xi}_{\tau \tau \tau \tau}^{K}\right\| \leq-c\left\|\boldsymbol{\xi}_{\tau \tau \tau \tau}^{K}\right\|^{2}+C \varepsilon^{-c \tau}+C \varepsilon^{-c \tau} h .
$$

By the methods leading to (8.23) we obtain $\left\|\boldsymbol{\xi}_{\tau \tau \tau \tau}^{K}\right\| \geq c h$, so that (8.66) yields

$$
h_{\tau} \leq-c h+C \varepsilon^{-c \tau}+C \varepsilon^{-c \tau} h \quad \Longrightarrow \quad|h| \leq C \varepsilon^{-c \tau} \quad \Longrightarrow \quad\left\|\boldsymbol{p}_{\tau \tau \tau}^{K}\right\|,\left\|\boldsymbol{w}_{\tau \tau \tau}^{K}\right\| \leq C \varepsilon^{-c \tau} .
$$

The substitution of the last inequality of (8.67) into (8.55) then yields $\left\|\boldsymbol{\xi}_{\tau \tau \tau}^{K}\right\| \leq C e^{-c \tau}$, so that (8.54) implies that

$$
\left\|\mathbf{v}_{\tau \tau \tau}^{K}\right\|,\left\|\mathbf{u}_{\tau \tau \tau}^{K}\right\|,\left\|\boldsymbol{p}_{s \tau \tau}^{K}\right\|,\left\|\boldsymbol{w}_{s \tau \tau}^{K}\right\| \leq C e^{-c \tau},
$$

whence

$$
\left|\boldsymbol{p}_{\tau \tau}^{K}\right|,\left|\boldsymbol{w}_{s \tau \tau}^{K}\right| \leq C e^{-c \tau}
$$

Clearly this approach gives exponential decay of $L^{2}$-norms of all multiple $\tau$-derivatives of $\boldsymbol{p}^{K}, \boldsymbol{w}^{K}$ (provided the data are smooth enough). The techniques exhibited above then give exponential decay of other derivatives.

Existence, regularity, and exponential decay of solutions of the initial-layer expansion. Fix an arbitrary $T>0$. The $L^{2}$-bounds (8.53), which are uniform in $K$ and $\tau$, give bounds on $\boldsymbol{\xi}_{\tau}^{K}(\cdot, \tau)$ in $H^{1}(0,1)$ uniform in $\tau$, so that $\boldsymbol{\xi}_{\tau}^{K}(\cdot, \tau)$ is bounded in $L^{2}\left(0, T, L^{2}(0,1)\right)$. A generalization [4, Lemma 11.33] of a compactness theorem of [6] implies that $\boldsymbol{\xi}_{\tau}^{K}(\cdot, \tau)$ lies in a compact subset of $C([0,1] \times[0, T])$. Consequences of this fact lead to a proof that $\left(\boldsymbol{p}^{K}, \boldsymbol{w}^{K}\right)$ converges uniformly to the unique weak solution $\left(\boldsymbol{p}_{0}^{\star}, \boldsymbol{w}_{0}^{\star}\right)$ of the initial-boundary-value problem for the leading terms of the initial-layer expansion. The details are omitted because they are special cases of those given in [4, Secs. 16-18]. 
Standard methods, such as the use of difference quotients [9, 13, 23], show that these solutions are classical, and in fact have as many derivatives as the data allow.

The uniform bounds (8.25) immediately yield the estimates

$$
\left|\boldsymbol{p}_{0}^{\star}(s, \tau)\right|,\left|\boldsymbol{w}_{0}^{\star}(s, \tau)\right| \leq C e^{-c \tau} .
$$

Now we specialize to our situation a theorem of [16, Sec. 5] as stated by [12, Thm. 1.6]:

Let $[0,1] \times[0, \infty) \supset \Omega \ni(s, \tau) \mapsto \varphi(s, \tau)$ be integrable on domain $\Omega$ and be approximated weakly by a sequence of $\nu$ times continuously differentiable functions $\varphi^{K}$ in the sense that $\int_{\Omega}\left[\varphi^{K}(s, \tau)-\varphi(s, \tau)\right] \psi(s, \tau) d s d \tau \rightarrow 0$ as $K \rightarrow \infty$ for every continuous function $\psi$ vanishing near the boundary of $\Omega$. If $\left\|\partial \varphi^{K} / \partial s^{\alpha} \partial \tau^{\beta}\right\|_{L^{2}(\Omega)} \leq C, \alpha+\beta=\nu$, then $\varphi$ has a distributional derivative $\partial \varphi / \partial s^{\alpha} \partial \tau^{\beta}$ satisfying $\left\|\partial \varphi / \partial s^{\alpha} \partial \tau^{\beta}\right\|_{L^{2}(\Omega)} \leq C$.

We identify $\varphi$ with components of $e^{c \tau} \boldsymbol{p}_{0}^{\star}$ and $e^{c \tau} \boldsymbol{w}_{0}^{\star}$ and their derivatives (with suitable $c$ 's). Then applying this theorem to (8.29), (8.30), (8.31), 8.47), (8.53), (8.58) (8.61) yields

$$
\begin{gathered}
\left\|\boldsymbol{p}_{0 \tau}^{\star}(s, \tau)\right\|,\left\|\boldsymbol{w}_{0 \tau}^{\star}(s, \tau)\right\|,\left\|\boldsymbol{p}_{0 \tau \tau}^{\star}(s, \tau)\right\|,\left\|\boldsymbol{w}_{0 \tau \tau}^{\star}(s, \tau)\right\|, \\
\left\|\boldsymbol{p}_{0 \tau \tau \tau}^{\star}(s, \tau)\right\|,\left\|\boldsymbol{w}_{0 \tau \tau \tau}^{\star}(s, \tau)\right\|,\left\|\boldsymbol{p}_{0 s}^{\star}(s, \tau)\right\|,\left\|\boldsymbol{w}_{0 s}^{\star}(s, \tau)\right\|, \\
\left\|\boldsymbol{p}_{0 s s}^{\star}(s, \tau)\right\|,\left\|\boldsymbol{w}_{0 s s}^{\star}(s, \tau)\right\|,\left\|\boldsymbol{p}_{0 s \tau}^{\star}(s, \tau)\right\|,\left\|\boldsymbol{w}_{0 s \tau}^{\star}(s, \tau)\right\|,\left\|\boldsymbol{p}_{0 s \tau \tau}^{\star}\right\|,\left\|\boldsymbol{w}_{0 s \tau \tau}^{\star}\right\|, \\
\left\|\mathbf{v}_{0 \tau}^{\star}(s, \tau)\right\|,\left\|\mathbf{u}_{0 \tau}^{\star}(s, \tau)\right\|,\left\|\mathbf{v}_{0 \tau \tau}^{\star}(s, \tau)\right\|,\left\|\mathbf{u}_{0 \tau \tau}^{\star}(s, \tau)\right\|, \\
\left\|\mathbf{v}_{0 s \tau}^{\star}(s, \tau)\right\|,\left\|\mathbf{u}_{0 s \tau}^{\star}(s, \tau)\right\|,\left\|\mathbf{v}_{0 \tau \tau \tau}^{\star}\right\|,\left\|\mathbf{u}_{0 \tau \tau \tau}^{\star}\right\| \\
\leq C e^{-c \tau}
\end{gathered}
$$

As in (8.39), there is a $\sigma_{j}(\tau)$ such that

$$
\mathbf{e}_{j} \cdot \boldsymbol{p}_{0 s}^{\star}(s, \tau)-\mathbf{e}_{j} \cdot \int_{0}^{1} \boldsymbol{p}_{0 s}^{\star}(\xi, \tau) d \xi=\mathbf{e}_{j} \cdot \int_{\sigma_{j}(\tau)}^{s} \boldsymbol{p}_{0 s s}^{\star}(\xi, \tau) d \xi
$$

with $\left|\int_{\sigma_{j}(\tau)}^{s} \boldsymbol{p}_{0 s s}^{\star}(\xi, \tau) d \xi\right| \leq\left[\int_{0}^{1}\left|\boldsymbol{p}_{0 s s}^{\star}(\xi, \tau)\right|^{2} d \xi\right]^{1 / 2}$ by the Cauchy-Bunyakovskiü-Schwarz inequality. Treating other functions likewise, we obtain from (8.71) that

$$
\left|\boldsymbol{p}_{0 s}^{\star}(s, \tau)\right|,\left|\boldsymbol{w}_{0 s}^{\star}(s, \tau)\right|,\left|\boldsymbol{p}_{0 \tau}^{\star}\right|,\left|\boldsymbol{w}_{0 \tau}^{\star}\right|,\left|\boldsymbol{p}_{0 \tau \tau}^{\star}\right|,\left|\boldsymbol{w}_{0 \tau \tau}^{\star}\right|,\left|\mathbf{v}_{0 \tau}^{\star}(s, \tau)\right|,\left|\mathbf{u}_{0 \tau}^{\star}(s, \tau)\right| \leq C e^{-c \tau} .
$$

Set

$$
\boldsymbol{n}^{\Delta}:=\boldsymbol{n}_{0}^{\star}-\boldsymbol{n}_{0}, \quad \mathbf{n}^{\Delta}:=\mathbf{n}_{0}^{\star}-\mathbf{n}_{0}, \quad \text { etc. }
$$

The classical equations (6.2) and (6.3) have the form

$$
\rho A \boldsymbol{p}_{0 \tau}^{\star}=\left(\boldsymbol{u}^{\circ} \times \boldsymbol{D}^{\circ}\right) \cdot \mathbf{n}^{\Delta}+\boldsymbol{D}^{\circ} \cdot\left[\mathbf{n}_{\dot{\boldsymbol{\xi}}}^{\triangleleft} \cdot \boldsymbol{\xi}_{0 s \tau}^{\star}+\mathbf{n}_{\bar{s}}^{\Delta}\right], \quad \text { etc. },
$$

where again the subscript $\bar{s}$ denotes the derivatives of $\hat{\mathbf{n}}$ and $\hat{\mathbf{m}}$ with respect to the $s$ appearing in $\boldsymbol{\xi}^{\circ}(s), \boldsymbol{\xi}_{0 t}(s, 0)$, and the last arguments of $\hat{\mathbf{n}}$ and $\hat{\mathbf{m}}$. The Mean-Value Theorem of (8.42) as in the treatment of (8.52) and the pointwise estimates already obtained show that all the terms in (8.75) except $\mathbf{n}_{\dot{\xi}}^{\star} \cdot \boldsymbol{\xi}_{0 s \tau}^{\star}$ and the invisible $\mathbf{m}_{\dot{\boldsymbol{\xi}}}^{\star} \cdot \boldsymbol{\xi}_{0 s \tau}^{\star}$ are pointwise exponentially decaying, whence we conclude from (8.14), (8.15), and (2.33) that

$$
\left|\mathbf{v}_{0 s \tau}^{\star}\right|,\left|\mathbf{u}_{0 s \tau}^{\star}\right|,\left|\boldsymbol{p}_{0 s s}^{\star}\right|,\left|\boldsymbol{w}_{0 s s}^{\star}\right| \leq C e^{-c \tau} .
$$

Thus we have pointwise decaying exponential bounds for all the derivatives that appear in the classical versions (8.75) of the equations of motion. 
To get further bounds we next differentiate (8.75) with respect to $\tau$ finding that all the terms in the resulting equations except $\mathbf{n}_{\dot{\xi}}^{\star} \cdot \boldsymbol{\xi}_{0 s \tau \tau}^{\star}$ and $\mathbf{m}_{\dot{\boldsymbol{\xi}}}^{\star} \cdot \boldsymbol{\xi}_{0 s \tau \tau}^{\star}$ are pointwise exponentially decaying, so that we likewise obtain

$$
\left|\mathbf{v}_{0 s \tau \tau}^{\star}\right|,\left|\mathbf{u}_{0 s \tau \tau}^{\star}\right|,\left|\boldsymbol{p}_{0 s s \tau}^{\star}\right|,\left|\boldsymbol{w}_{0 s s \tau}^{\star}\right| \leq C e^{-c \tau} .
$$

Such pointwise estimates can be obtained for any derivatives of $\boldsymbol{p}_{0}^{\star}, \boldsymbol{w}_{0}^{\star}, \boldsymbol{\xi}_{0}^{\star}$ provided the data are smooth enough. Indeed, the derivatives of $\boldsymbol{p}_{0}^{\star}$ and $\boldsymbol{w}_{0}^{\star}$ satisfy linear parabolic systems with the dissipativity condition (2.32) responsible for the desired exponential decay. Likewise, the higher-order terms of the initial-layer expansion each satisfy linear parabolic systems, and the same methods ensure the exponential decay of their solutions and derivatives of their solutions. Such derivatives of the variables of any order of the initial-layer expansion intervene in equations of higher order. A virtually identical approach produces exponential decay of these variables and their derivatives. We omit the details.

9. Justification. We give just the barest hint of the justification, which has the same structure as [21, Sec. 5]. Conditions (5.1) 1 , (7.1), (7.4) imply that

$$
\begin{aligned}
\varepsilon^{-1} & \partial_{\tau} \boldsymbol{v}_{\mathrm{A}}^{H}(s, \varepsilon \tau ; \varepsilon)-\partial_{s} \boldsymbol{p}_{\mathrm{A}}^{H}(s, \varepsilon \tau ; \varepsilon) \\
= & \sum_{j=0}^{H}\left[\boldsymbol{v}_{j t}(s, \varepsilon \tau)-\boldsymbol{p}_{j s}(s, \varepsilon \tau)\right] \frac{\varepsilon^{j}}{j !}+\sum_{j=0}^{k-1}\left[\boldsymbol{v}_{j \tau}^{\star}(s, \tau) \frac{\varepsilon^{j}}{(j+1) !}-\boldsymbol{p}_{j s}^{\star}(s, \tau) \frac{\varepsilon^{j}}{j !}\right]-\boldsymbol{p}_{k s}^{\star}(s, \tau) \frac{\varepsilon^{k}}{k !} \\
= & \sum_{j=0}^{k-1}\left[\frac{\boldsymbol{v}_{j \tau}^{\star}(s, \tau)}{j+1}-\boldsymbol{p}_{j s}^{\star}(s, \tau)\right] \frac{\varepsilon^{j}}{j !}-\boldsymbol{p}_{k s}^{\star}(s, \tau) \frac{\varepsilon^{k}}{k !}=-\boldsymbol{p}_{k s}^{\star}(s, \tau) \frac{\varepsilon^{k}}{k !} .
\end{aligned}
$$

We know that there exists a regular solution $\boldsymbol{v}, \ldots$ of the full problem. Let

$$
\boldsymbol{v}_{\mathrm{E}}^{H}(s, t ; \varepsilon):=\boldsymbol{v}(s, t ; \varepsilon)-\boldsymbol{v}_{\mathrm{A}}^{H}(s, t ; \varepsilon), \quad \text { etc. }
$$

Then (9.1) implies that

$$
\partial_{t} \boldsymbol{v}_{\mathrm{E}}^{H}-\partial_{s} \boldsymbol{p}_{\mathrm{E}}^{H}=\partial_{s} \boldsymbol{p}_{H}^{\star}(s, \tau) \frac{\varepsilon^{H}}{H !} .
$$

An energy estimate using (9.3) and its companion compatibility equation followed by a Gronwall inequality leads to

$$
\int_{0}^{1}\left[\boldsymbol{p}_{\mathrm{E}}^{H}(s, \tau ; \varepsilon)\right]^{2} d s \leq C(H, T) \varepsilon^{2 H-1}
$$

where $C(H, T)$ is a typical positive constant depending only on $H$, the time interval $[0, T]$, and the data. (Cf. the paragraph following (8.6).) The power $2 H-1$ on $\varepsilon$ is inadequate for the error terms in (3.2). To boost this power, we use (9.4) to obtain

$$
\begin{aligned}
\int_{0}^{1}\left[\boldsymbol{p}_{\mathrm{E}}^{H}(s, \tau ; \varepsilon)\right]^{2} d s & =\int_{0}^{1}\left[\boldsymbol{p}-\boldsymbol{p}_{\mathrm{A}}^{H+1}+\boldsymbol{p}_{\mathrm{A}}^{H+1}-\boldsymbol{p}_{\mathrm{A}}^{H}\right]^{2} d s \\
& \leq 2 \int_{0}^{1}\left[\boldsymbol{p}_{\mathrm{E}}^{H+1}\right]^{2} d s+2 \int_{0}^{1}\left[\boldsymbol{p}_{\mathrm{A}}^{H+1}-\boldsymbol{p}_{\mathrm{A}}^{H}\right]^{2} d s \leq C(H, T) \varepsilon^{2 H+1} .
\end{aligned}
$$


The application of elementary embedding and interpolation lemmas and the use of a priori bounds from [4] ultimately lead to the pointwise estimate

$$
\left|\partial_{s} \boldsymbol{p}_{\mathrm{E}}^{H}(s, \tau ; \varepsilon)\right| \leq C(H, T) \varepsilon^{(H+1) / 4} .
$$

The repeated use of the telescoping method (9.5) yields

$$
\left|\partial_{s} \boldsymbol{p}_{\mathrm{E}}^{H}(s, \tau ; \varepsilon)\right| \leq C(H, T) \varepsilon^{H+1}
$$

(provided the data have far more regularity than that used to justify (9.5)).

10. Comments. There are many variants of the problem of Section 2 that our methods can handle: Both the deformable rod and the rigid attachments could be subjected to time-dependent forcing [21. The boundary conditions at each end could be replaced with a variety of others: E.g., the rigid body or a collection of rigid bodies could be attached anywhere on the rod (in which case it might be feasible to regard the deformable rod as a collection of deformable rods joined at the attachments where there would be transmission conditions [5], and the attachments could be maintained by hinges rather than by welds. As long as there is a strong dissipative mechanism (giving the system a parabolic character), like the second condition of (2.17), it seems likely that our methods could handle a wide variety of constitutive assumptions, e.g., those with memory effects. (On the other hand, materials with memory lacking such strong dissipation 8 might require very different techniques and exhibit very different behavior. In this respect, it is interesting to note that the reduced problem exhibits memory effects.)

The lower-order term $\boldsymbol{r}_{s} \times \boldsymbol{n}$ in the moment balance (2.22) does not behave like a typical lower-order term, as we have seen in the treatment of energy equations. It may be a consequence of the constraint that $\boldsymbol{D}$ lie in $\mathrm{SO}(3)$. Its presence complicates the study of exponential decay, which would be straightforward for abstract systems with no lower-order terms and no rotations $\boldsymbol{D}$.

We could have carried out our Galerkin approximation with eigenvalues $\alpha_{k}$ and eigenfunctions $\boldsymbol{x}_{k}$ for

$$
\boldsymbol{x}_{k s s}+\alpha_{k} \rho A(s) \boldsymbol{x}_{k}=\boldsymbol{o}, \quad \boldsymbol{x}_{k}(0)=\boldsymbol{o}=\boldsymbol{x}_{k}(1),
$$

and with eigenvalues $\beta_{k}$ and eigenfunctions $\boldsymbol{y}_{k}$ for

$$
\boldsymbol{y}_{k s s}+\beta_{k} \rho \boldsymbol{I}_{0} \cdot \boldsymbol{y}_{k}=\boldsymbol{o}, \quad \boldsymbol{y}_{k}(0)=\boldsymbol{o}=\boldsymbol{y}_{k}(1)
$$

where $\left(\rho \boldsymbol{I}_{0}\right)(s):=\boldsymbol{D}^{\circ}(s) \cdot \rho \mathbf{I}(s) \cdot \mathbf{D}^{\circ}(s)^{\top}$. Though these vectorial eigenfunctions account for the inertia of the rod, they complicate the analysis.

There is a hierarchy of rod theories, with more refined theories accounting for further kinematic effects, such as thickness changes. Our methods could readily treat problems for such theories. Some of our methods could be used for problems for shells and 3dimensional bodies, which seem to require results on quasilinear elliptic systems to treat the regular expansion.

Acknowledgment. We are grateful to Abner Salgado for helpful comments. The research reported here was supported in part by a grant from the NSF. 


\section{REFERENCES}

[1] S. S. Antman, The paradoxical asymptotic status of massless springs, SIAM J. Appl. Math. 48 (1988) 1319-1334. MR968832(90b:35194)

[2] S. S. Antman, Nonlinear Problems of Elasticity, 2nd edition, Springer, 2005. MR2132247 (2006e:74001)

[3] S.S. Antman, R.S. Marlow, and C.P. Vlahacos, The complicated dynamics of heavy rigid bodies attached to light deformable rods, Quart. Appl. Math. 56 (1998) 431-460. MR1637036 (99e:73068)

[4] S.S. Antman and T.I. Seidman, The parabolic-hyperbolic system governing the spatial motion of nonlinearly viscoelastic rods, Arch. Rational Mech. Anal. 175 (2005) 85-150. MR2106258 (2006b:74015)

[5] S. S. Antman and J. P. Wilber, The asymptotic problem for the springlike motion of a heavy piston in a viscous gas, Quart. Appl. Math. 65 (2007) 471-498. MR2354883 (2009g:76128)

[6] J. P. Aubin, Un théorème de compacité, C. R. Acad. Sci., Paris 265 (1963) 5042-5045. MR0152860 $(27: 2832)$

[7] M. F. Beatty, Finite amplitude oscillations of a simple rubber support system, Arch. Rational Mech. Anal. 83 (1983) 195-219. MR701902 (84g:73039)

[8] C. M. Dafermos and J. A. Nohel, Energy methods for nonlinear hyperbolic differential equations, Comm. P.D.E.s 4 (1979) 219-278. MR522712 (80b:45018)

[9] A. Friedman, Partial Differential Equations of Parabolic Type, Prentice-Hall, 1964. MR0181836 $(31: 6062)$

[10] G. H. Handelman and J. B. Keller, Small vibrations of a slightly stiff pendulum. Proc. 4th U.S. Nat. Congr. Appl. Mech., Vol. 1, Amer. Soc. Mech. Engrs., 1962, pp. 195-202. MR0153150 (27:3119)

[11] P. Krishnaprasad and J.E. Marsden, Hamiltonian structures and stability for rigid bodies with flexible attachments, Arch. Rational Mech. Anal. 98 (1987) 71-93. MR866725 (87m:58084)

[12] O. A. Ladyženskaja, Mixed Problems for Hyperbolic Equations (in Russian), GITTL, Moscow, 1953. MR0071631(17:160c)

[13] O. A. Ladyženskaja, V.A. Solonnikov, and N. N. Ural'ceva, Linear and Quasilinear Equations of Parabolic Type, Translations of Mathematical Monographs, vol. 23, Amer. Math. Soc., 1967. MR 0241822(39:3159b)

[14] J.-L.Lions, Quelques méthodes de résolution des problèmes aux limites non linéaires, Dunod, Gauthier-Villars, 1969. MR0259693(41:4326)

[15] D. R. Smith, Singular Perturbation Theory, Cambridge, 1985. MR812466 (87d:34001)

[16] S. L. Sobolev, Applications of Functional Analysis in Mathematical Physics, Amer. Math. Soc., 1963. MR0165337 (29:2624)

[17] M. Wiegner, On the asymptotic behaviour of solutions of nonlinear parabolic equations, Math. Z. 188 (1984) 3-22. MR767358 (86b:35017)

[18] J. P. Wilber, Absorbing balls for equations modeling nonuniform deformable bodies with heavy rigid attachments, J. Dyn. Diff. Eqs. 19 (2002) 855-887. MR1940106 (2003j:37139)

[19] J. P. Wilber, Invariant manifolds describing the dynamics of a hyperbolic-parabolic equation from nonlinear elasticity, Dyn. Systems 21 (2006) 465-490. MR2273689 (2007m:37197)

[20] J. P. Wilber and S. S. Antman, Global attractors for a degenerate partial differential equation from nonlinear viscoelasticity, Physica D 150 (2001) 179-208. MR1820734(2001m:74013)

[21] S.-C. Yip, S. S. Antman, and M. Wiegner, The motion of a particle on a light viscoelastic bar: Asymptotic analysis of the quasilinear parabolic-hyperbolic equation, J. Math. Pures Appl. $\mathbf{8 1}$ (2002) 283-309. MR1967351(2004k:35378)

[22] W. Weaver, Jr., S. P. Timoshenko, and D. H. Young, Vibration Problems in Engineering, 5th edition, Wiley, 1990.

[23] E. Zeidler, Nonlinear Functional Analysis and its Applications, Vol. IIB, Springer-Verlag, New York, 1990. MR:1033498(91b:47002) 